\title{
Ultrafast Spectroscopic Signatures of Coherent Electron-Transfer Mechanisms in a Transition Metal Complex
}

\author{
Zhenkun Guo, Paul G. Giokas, Thomas P. Cheshire, Olivia F. Williams, David J. Dirkes, Wei You, \\ and Andrew M. Moran* \\ Department of Chemistry, University of North Carolina at Chapel Hill, Chapel Hill, North Carolina 27599, United States
}

\begin{abstract}
The prevalence of ultrafast electron-transfer processes in lightharvesting materials has motivated a deeper understanding of coherent reaction mechanisms. Kinetic models based on the traditional (equilibrium) form of Fermi's Golden Rule are commonly employed to understand photoinduced electron-transfer dynamics. These models fail in two ways when the electron-transfer process is fast compared to solvation dynamics and vibrational dephasing. First, electron-transfer dynamics may be accelerated if the photoexcited wavepacket traverses the point of degeneracy between donor and acceptor states in the solvent coordinate. Second, traditional kinetic models fail to describe electron-transfer transitions that yield products which undergo coherent nuclear motions. We address the second point in

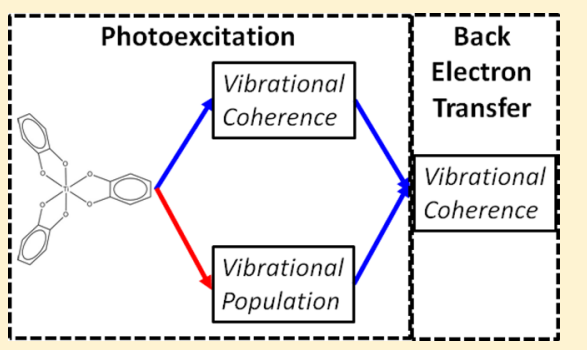
this work. Transient absorption spectroscopy and a numerical model are used to investigate coherent back-electron-transfer mechanisms in a transition metal complex composed of titanium and catechol, $\left[\mathrm{Ti}(\mathrm{cat})_{3}\right]^{2-}$. The transient absorption experiments reveal coherent wavepacket motions initiated by the back-electron-transfer process. Model calculations suggest that the vibrationally coherent product states may originate in either vibrational populations or coherences of the reactant. That is, vibrational coherence may be produced even if the reactant does not undergo coherent nuclear motions. The analysis raises a question of broader significance: can a vibrational population-to-coherence transition (i.e., a nonsecular transition) accelerate electron-transfer reactions even when the rate is slower than vibrational dephasing?
\end{abstract}

\section{INTRODUCTION}

Knowledge of coherent chemical reaction mechanisms has motivated a large body of work in the past few decades. ${ }^{1-8}$ Interest in the role of vibronic coherence in electron-transfer processes can be traced back to the earliest experimental studies of femtosecond dynamics in the bacterial photosynthetic reaction center. ${ }^{1,2}$ This study was followed by numerous observations of similar behaviors in other biological systems; examples include photoinduced isomerization of bacteriorhodopsin ${ }^{3}$ and photodissociation in myoglobin. ${ }^{5}$ Recent advances in femtosecond laser spectroscopies have enabled more detailed investigations of coherent electron-transfer mechanisms in mixed valence systems, ${ }^{9}$ photosynthetic proteins, ${ }^{10}$ polymerfullerene blends, ${ }^{11}$ and molecule-semiconductor interfaces. ${ }^{12,13}$ A commonality in these systems is that electron transfer takes place on a time scale that is shorter than vibrational dephasing and/or solvation dynamics. Traditional second-order models should not be applied in this nonequilibrium regime. ${ }^{14-17}$ Recurrences of the donor at the transition state may give rise to periodic bursts in the acceptor's population in some cases. ${ }^{1,2,6,10,11,18}$ An understanding of such coherent reaction dynamics holds implications for hot electron-transfer processes, which can boost solar energy conversion efficiency if properly harnessed. ${ }^{19-22}$

In this paper, femtosecond transient absorption spectroscopy is used to investigate the influence of vibronic coherence on electron transfer in a transition metal complex in which a titanium atom is bonded to three catechol ligands, $\left[\mathrm{Ti}(\mathrm{cat})_{3}\right]^{2-}$. Strong interactions between catechol and titanium give rise to a charge-transfer electronic resonance near $400 \mathrm{~nm} .{ }^{23}$ Following light-induced electron transfer from catechol to titanium, the strong titanium-catechol interactions also promote fast backelectron-transfer (BET) from titanium to catechol. ${ }^{24}$ The welldefined nature of $\left[\mathrm{Ti}(\mathrm{cat})_{3}\right]^{2-}$ is leveraged to understand how the $\mathrm{BET}$ process initiates coherent nuclear motion in the product state. The charge-transfer nature of the electronic resonance of $\left[\mathrm{Ti}(\mathrm{cat})_{3}\right]^{2-}$ is essential for this investigation, because it guarantees significant projections of FranckCondon active modes onto the reaction coordinate. ${ }^{6,25-29}$ With inspiration from earlier work, ${ }^{6,27-29}$ we use normal mode displacements determined with resonance Raman spectroscopy to parametrize a nonequilibrium model that incorporates coherent BET mechanisms.

Photoexcitation and BET are decomposed into four "pathways" in Figure 1. Traditional (equilibrium) secondorder kinetic models describe the pathway connected by red arrows for thermalized excited states. The other three possibilities involve coherent nuclear motion before and/or after the BET process. Coherent nuclear motion is directly initiated by light absorption in most of the previous

Received: April 28, 2016

Revised: June 24, 2016

Published: June 30, 2016 


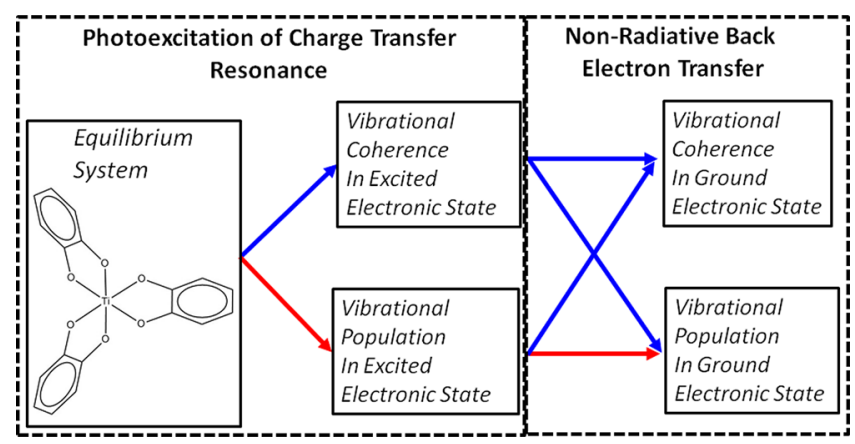

Figure 1. Photoinduced back-electron-transfer dynamics of [Ti$\left.(\mathrm{cat})_{3}\right]^{2-}$ described with four potential pathways. The pathway connected by red arrows can be described with traditional models if a Boltzmann distribution of vibrational quanta is established before back-electron-transfer; however, a nonequilibrium electron-transfer model is required if this condition is not satisfied. The pathways involving vibrational coherences contribute if back-electron-transfer is faster than vibrational dephasing.

experimental studies discussed above (i.e., a stimulated Raman process). Bursts of population flow into the product state may be observed in such cases, and the wavepacket can retain coherence in the product state (i.e., a vibronic coherencetransfer transition). ${ }^{1,2,6,10,11,18}$ It is also possible for vibrational coherence to be initiated in the product state if the reactant does not undergo coherent nuclear motion. Fleming and Jean emphasized this point in an earlier theoretical study based on a density matrix approach. ${ }^{30}$ The two pathways in which BET initiates vibrational coherence in the product are of primary interest in this work (i.e., paths ending on top in Figure 1).

In previous work, we showed that a fourth-order perturbative model can be used to simulate time-coincident electron-transfer and solvation dynamics under many of the same approximations made in Marcus' theory. ${ }^{31}$ The model takes inspiration from an earlier nonequilibrium version of Fermi's golden rule in which explicit field-matter interactions were not used to establish the initial condition. ${ }^{32}$ Similarly motivated models for nonequilibrium electron transfer ${ }^{33}$ and nonsecular transitions between vibrational states ${ }^{34}$ have been presented in recent literature. Our model is distinct in that it is specific to an electron-transfer process, the initial condition is determined by explicit field-matter interactions, and multiple promoting modes are treated. In earlier work, we modeled the behavior of a system initiated by light absorption using experimentally accessible parameters such as the solvation time and absorbance line width. ${ }^{31}$ The fourth-order model revealed large discrepancies with Marcus' theory in regimes, where the photoinitiated wavepacket traverses the point of intersection between diabatic surfaces. ${ }^{31}$ This earlier model is applicable to systems with weak vibronic activity; however, we show in this work that the addition of quantized vibrational levels is relatively straightforward and will support a description of the coherent pathways in Figure 1.

\section{MODEL FOR SPECTROSCOPY AND DYNAMICS}

In this section, we develop a model for transient absorption spectroscopy that incorporates the four types of BET transitions defined in Figure 1. Similar to previous resonance Raman studies, $6,27-29$ the model is developed under the assumption that the optical response and BET process depend on the same parameters because of the charge-transfer nature of the electronic resonance. The model will be parametrized using a spectral fitting approach for which key equations are summarized at the end of this section.

IIA. Hamiltonian. We employ a reduced description in which the Hamiltonian is partitioned into three components: the system $\left(H_{\text {sys }}\right)$, bath $\left(H_{\text {bath }}\right)$, and system-bath interaction $\left(H_{\text {sys-bath }}\right)$. The three operators may be written as

$$
\begin{aligned}
& H_{\text {sys }}=|\mathrm{g}\rangle\left\langle\mathrm{g}\left|\sum_{m}\right| m\right\rangle\left\langle m \mid\left(E_{\mathrm{g}}+E_{m}\right)+\mathrm{e}\right\rangle\left\langle\mathrm{e}\left|\sum_{n}\right| n\right\rangle\langle n|\left(E_{\mathrm{e}}+E_{n}\right) \\
& H_{\text {bath }}=\frac{1}{2} \sum_{i}\left[\frac{p_{i}^{2}}{\mu_{i}}+\mu_{i} \omega_{i}{ }^{2} q_{i}^{2}\right][|\mathrm{g}\rangle\langle\mathrm{g}|+| \mathrm{e}\rangle\langle\mathrm{el}]
\end{aligned}
$$

and

$$
H_{\text {sys-bath }}=|g\rangle Q_{\mathrm{g}, c}\langle\mathrm{~g}|+| \mathrm{e}\rangle Q_{\mathrm{e}, c}\langle\mathrm{e}|
$$

The dynamics of interest in this work can be simulated by treating only the ground $(\mathrm{g})$ and lowest energy excited (e) electronic states of the system if we assume that BET is fast compared to transitions between excited states. The summations in $H_{\text {sys }}$ are carried out over dummy indices, $m$ and $n$, that represent quantized vibrational levels of the system. Throughout this paper, the indices $m, l$, and $u$ will be associated with the ground electronic state, whereas $n, k$, and $v$ will correspond to the excited electronic state. In the harmonic approximation, the basis vectors are direct products, $|m\rangle=\prod_{\alpha} \mid$ $\left.N_{\alpha m}\right\rangle$, where $N_{\alpha m}$ is the number of vibrational quanta in normal mode $\alpha$ for basis vector $m$. The bath consists of displaced harmonic oscillators for which the associated variables are written in lower case $\left(\mu_{i}, \omega_{i}\right.$, and $\left.q_{i}\right) .^{14-16}$ The collective primary oscillator coordinates in $H_{\text {sys-bath }}$ are coupled to the secondary harmonic modes in $H_{\text {bath }}$ according to $Q_{\mathrm{g}, c}=$ $\sum_{i} \mu_{i} \omega_{i}^{2} d_{\mathrm{g}, i} q_{i}$, where $d_{\mathrm{g}, i}$ is the displacement of mode $i$ for the ground electronic state; an analogous formula can be written for the excited electronic state. In the present reduced description, the bath coordinates are never explicitly parametrized, because a trace is carried out over the states of the bath. $^{14}$

Stochastic fluctuations in the energy levels of the system are handled using a time-correlation function formalism for the collective coordinates. The cumulant expansion approach may be employed, because the bath is taken to be harmonic. ${ }^{14-16}$ In the overdamped limit, time-correlation functions between the collective Brownian oscillator coordinates are related to the second term in a cumulant expansion by ${ }^{14}$

$$
\gamma(t)=\left(\frac{2 \lambda k_{\mathrm{B}} T}{\hbar^{2} \Lambda^{2}}-\mathrm{i} \frac{\lambda}{\hbar \Lambda}\right)[\exp (-\Lambda t)+\Lambda t-1]
$$

where $\Lambda^{-1}$ is the time scale of nuclear relaxation and $\lambda$ is the reorganization energy. The reorganization energy of the bath is related to the variance of fluctuations in the energy gap between ground and excited states by

$$
\lambda=\frac{\Delta^{2}}{2 k_{\mathrm{B}} T}
$$

The assumption of an overdamped primary oscillator coordinate is justified for systems in which vibronic structure in the absorbance spectrum is not resolved (i.e., Gaussian absorbance line shapes). Both electron transfer and light 
absorbance are governed by the same reorganization energy in systems that possess charge-transfer resonances. ${ }^{6,25-29}$

Perturbative interactions with the system occur with both laser pulses, $\vec{E} \cdot \vec{\mu}_{\mathrm{eg}}$, and with the donor-acceptor coupling, $V_{\mathrm{eg}}$. Explicit forms of the donor-acceptor coupling and electronic transition dipole are not required, because they enter the models as constant prefactors under the Condon approximation (this will be shown below). The system-bath coupling is not perturbative. Therefore, the model can be used to treat systems with large reorganization energies and broad spectroscopic line widths.

IIB. Model for Transient Absorption Signals. The model presented in this section establishes transient absorption signatures of the nonequilibrium pathways in Figure 1. Both populations and coherences between vibrational states can be treated by extending the fourth-order perturbative model for electron transfer developed in earlier work. ${ }^{31,35}$ The system undergoes BET between pump and probe events in the signal component of interest. Overall, the process can be described as a sequence of six perturbative interactions. The formulas can be greatly simplified by invoking a "doorway-window" picture in which the signal is written as a product of wavepackets associated with excitation and detection. Such models have been used extensively in descriptions of transient absorption experiments. $^{14}$

The doorway function incorporates dynamics that take place in the $t_{1}, t_{2}$, and $t_{3}$ time intervals shown in Figure 2. In this

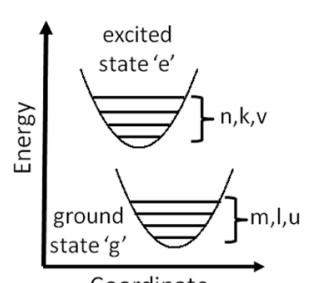

Coordinate
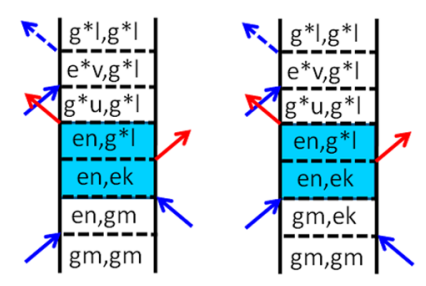

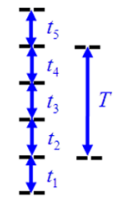

Figure 2. Nonlinearities associated with the hot ground-state signal component obtained by incorporating two field-matter interactions before and after the back-electron-transfer process. The time intervals, $t_{i}$ separate perturbative interactions with an external electric field, $\hat{H}_{\text {rad-mat }}$ (blue arrows), and the donor-acceptor coupling, $\hat{V}$ (red arrows). The indices $g$ and e represent ground and excited states of $\left[\mathrm{Ti}(\mathrm{cat})_{3}\right]^{2-}$, whereas the vibrational levels are represented by dummy indices $(m, n, k, l, u, v)$. The indices $m, l$, and $u$ are associated with the ground electronic state, whereas $n, k$, and $v$ correspond to the excited electronic state. The nonradiative transition from state e to state $g$ is referred to as "back-electron-transfer" (i.e., the events shaded in blue).

approach, we must assume that the dynamics are uncorrelated with those in the $t_{4}$ and $t_{5}$ time intervals. The assumption is good for systems in aqueous solutions, which possess fast solvation times. ${ }^{36}$ The two components of the doorway function can be written as

$$
\begin{aligned}
\phi_{1}\left(t_{2}\right)= & 2\left|\mu_{\mathrm{eg}}\right|^{2}\left|V_{e g}\right|^{2} \hbar^{4} \sum_{m n k l u} B_{m}\langle n \mid m\rangle\langle k \mid m\rangle\langle n \mid u\rangle\langle k \mid l\rangle \\
& \times \operatorname{Re}\left\{\int_{0}^{\infty} \mathrm{d} t_{1} \int_{0}^{\infty} \mathrm{d} t_{2} \int_{0}^{\infty} \mathrm{d} t_{3}\right. \\
& \times \exp \left[-\mathrm{i}\left(\omega_{\mathrm{eg}} t_{1}+\omega_{n m} t_{1}+\omega_{n k} t_{2}-\mathrm{i} \Gamma_{n k} t_{2}+\omega_{\mathrm{eg}} t_{3}+\omega_{n l} t_{3}\right)\right. \\
& \left.\left.-\frac{1}{2} \Delta^{2}\left(t_{1}{ }^{2}+t_{3}{ }^{2}\right)-f\left(t_{1}, t_{2}, t_{3}\right)\right] E^{*}\left(t^{\prime}-t_{1}\right) E\left(t^{\prime}\right)\right\}
\end{aligned}
$$

and

$$
\begin{aligned}
\phi_{2}\left(t_{2}\right)= & 2\left|\mu_{\mathrm{eg}}\right|^{2}\left|V_{\mathrm{eg}}\right|^{2} \hbar^{4} \sum_{m n k l u} B_{m}\langle n \mid m\rangle\langle k \mid m\rangle\langle n \mid u\rangle\langle k \mid l\rangle \\
& \times \operatorname{Re}\left\{\int_{0}^{\infty} \mathrm{d} t_{1} \int_{0}^{\infty} \mathrm{d} t_{2} \int_{0}^{\infty} \mathrm{d} t_{3}\right. \\
& \times \exp \left[-\mathrm{i}\left(-\omega_{\mathrm{eg}} t_{1}+\omega_{m k} t_{1}+\omega_{n k} t_{2}-\mathrm{i} \Gamma_{n k} t_{2}+\omega_{\mathrm{eg}} t_{3}+\omega_{n l} t_{3}\right)\right. \\
& \left.\left.-\frac{1}{2} \Delta^{2}\left(t_{1}{ }^{2}+t_{3}{ }^{2}\right)+f^{*}\left(t_{1}, t_{2}, t_{3}\right)\right] E^{*}\left(t^{\prime}-t_{1}\right) E\left(t^{\prime}\right)\right\}
\end{aligned}
$$

where

$$
f\left(t_{1}, t_{2}, t_{3}\right)=\gamma^{*}\left(t_{2}\right)-\gamma^{*}\left(t_{2}+t_{3}\right)-\gamma\left(t_{1}+t_{2}\right)+\gamma\left(t_{1}+t_{2}+t_{3}\right)
$$

In eqs 6 and 7, we use the convention of ref 37, where vibrational levels of the excited state are written in the bra of the inner products.

The five nested summations over vibrational levels will be computationally expensive to evaluate for a system with multiple vibrational modes. Analytic integration can be accomplished with inspiration from earlier descriptions of transient absorption spectroscopies. ${ }^{14,38}$ To begin, the effect of finite laser bandwidth is incorporated by introducing the integration variable, $t^{\prime}=\tau-t_{2}$. This approximation is justified when the temporal width of the laser pulse is short compared to the time scale of the nuclear dynamics. In addition we make the "short-time approximation" for the linear combination of line broadening functions, $\gamma(t)$, in $f\left(t_{1}, t_{2}, t_{3}\right)$. Similar approaches were used to described electron transfer and internal conversion in earlier work. ${ }^{31,35}$ The two functions are then written as

$$
\begin{aligned}
\phi_{1}(\tau)= & 2\left|\mu_{\mathrm{eg}}\right|^{2}\left|V_{\mathrm{eg}}\right|^{2} \hbar^{4} \sum_{m n k l u} B_{m}\langle n \mid m\rangle\langle k \mid m\rangle\langle n \mid u\rangle\langle k \mid l\rangle \\
& \times \operatorname{Re}\left\{\int_{0}^{\infty} \mathrm{d} t_{1} \int_{-\infty}^{\infty} \mathrm{d} t^{\prime} \int_{0}^{\infty} \mathrm{d} t_{3}\right. \\
& \times \exp \left[-\mathrm{i}\left(\omega_{\mathrm{eg}} t_{1}+\omega_{n m} t_{1}+\omega_{n k} \tau-\mathrm{i} \Gamma_{n k} \tau+\omega_{\mathrm{eg}} t_{3}+\omega_{n l} t_{3}\right)\right. \\
& \left.\left.-\frac{1}{2} \Delta^{2}\left(t_{1}{ }^{2}+t_{3}{ }^{2}\right)-h\left(t_{1}, \tau, t_{3}\right)\right] E^{*}\left(t^{\prime}-t_{1}\right) E\left(t^{\prime}\right)\right\}
\end{aligned}
$$

and

$$
\begin{aligned}
\phi_{2}(\tau)= & 2\left|\mu_{\mathrm{eg}}\right|^{2}\left|V_{\mathrm{eg}}\right|^{2} \hbar^{4} \sum_{m n k l u} B_{m}\langle k \mid m\rangle\langle n \mid m\rangle\langle n \mid u\rangle\langle k \mid l\rangle \\
& \times \operatorname{Re}\left\{\int_{0}^{\infty} \mathrm{d} t_{1} \int_{-\infty}^{\infty} \mathrm{d} t^{\prime} \int_{0}^{\infty} \mathrm{d} t_{3}\right. \\
& \times \exp \left[-\left(-\omega_{\mathrm{eg}} t_{1}+\omega_{m k} t_{1}+\omega_{n k} \tau-\mathrm{i} \Gamma_{n k} \tau+\omega_{\mathrm{eg}} t_{3}+\omega_{n l} t_{3}\right)\right. \\
& \left.\left.-\frac{1}{2} \Delta^{2}\left(t_{1}^{2}+t_{3}^{2}\right)+h^{*}\left(t_{1}, \tau, t_{3}\right)\right] E^{*}\left(t^{\prime}-t_{1}\right) E\left(t^{\prime}\right)\right\}
\end{aligned}
$$

where

$$
h\left(t_{1}, \tau, t_{3}\right)=\mathrm{i} \frac{2 \lambda}{\hbar}[\exp (-\Lambda \tau)-1] t_{3}+\Delta^{2} \hbar^{2} \exp (-\Lambda \tau) t_{1} t_{3}
$$

In addition, a Gaussian laser spectrum will be assumed for convenience,

$$
E(t)=\xi_{L} \exp \left(-\mathrm{i} \omega_{\mathrm{L}} t-w_{\mathrm{L}}{ }^{2} t^{2} / 2 \hbar^{2}\right)
$$

Equations 9 and 10 set up the description in a way that parallels an earlier formulation of transient absorption signals. $^{14,38}$ The key difference in the present case is that quantized vibrational levels have been incorporated into the system Hamiltonian (i.e., underdamped intramolecular modes are not relegated to the bath). ${ }^{14}$ The present formulas assume 
the same mathematical form as the earlier model if vibrational levels are removed from the first time interval between fieldmatter interactions, $\omega_{\mathrm{ge}} t_{1}+\omega_{m n} t_{1} \approx \omega_{\mathrm{ge}} t_{1}$. This is a good approximation if the absorbance spectrum has a broad Gaussian shape (i.e., vibronic progression is unresolved). The sum of eqs 9 and 10 then yields

$$
D(\tau)=\sum_{m n k l u} D_{n k \rightarrow l u}(\tau)
$$

where

$$
\begin{aligned}
& D_{n k \rightarrow u l}(\tau)= \pi^{3 / 2}\left|\xi_{\mathrm{L}} \mu_{\mathrm{eg}}\right|^{2}\left|V_{\mathrm{eg}}\right|^{2} \hbar w_{\mathrm{L}}^{2}\left(\Delta^{2}+w_{\mathrm{L}}^{2}\right) \alpha^{2}(\tau) \\
& \times B_{m}\langle n \mid m\rangle\langle k \mid m\rangle\langle n \mid u\rangle\langle k \mid l\rangle \\
& \times \exp \left[-\frac{\hbar^{2}\left(\omega_{L}-\omega_{e g}\right)^{2}}{4 \lambda k_{\mathrm{B}} T}\right] \exp \left[-\frac{\Omega^{2}(\tau)}{2 \alpha^{2}(\tau)}\right] \\
& \times \cos \left(\omega_{n k} \tau\right) \exp \left(-\Gamma_{n k} \tau\right) \\
& \Omega(\tau)=\Delta G_{\mathrm{eg}}^{0}+\lambda-\hbar \omega_{n l}+\exp (-\Lambda \tau)\left[-\hbar \omega_{\mathrm{L}}-\Delta G_{\mathrm{eg}}^{0}\right. \\
&\left.-\lambda+\hbar \omega_{n l}\right]
\end{aligned}
$$

and

$$
\alpha^{2}(\tau)=\Delta^{2}\left[1-\frac{\Delta^{2}}{\Delta^{2}+w_{\mathrm{L}}^{2}} \exp (-2 \Lambda \tau)\right]
$$

The laser spectrum is assumed to be narrower than the absorbance line width in $\Omega(\tau)$, because this approximation is appropriate for the experiments described in section IV. The magnitude of the free energy gap, $\Delta G_{\mathrm{eg}}^{0}$, is equal to the difference between the peak of the absorbance spectrum, $\omega_{\text {eg }}$, and the solvent reorganization energy, $\lambda$ (i.e., $\Delta G_{\mathrm{eg}}^{0}$ corresponds to the "electronic origin").

We resort to an empirical approach in writing the line shape of the window function. The signal associated with the "hot ground state" induced by BET undergoes a vibrational cooling process following $\mathrm{BET}$, which increases the electronic resonance frequency. An explicit treatment of such vibrational cooling mechanisms is beyond the scope of this paper. Therefore, we take the hot ground state into account with empirical functions, $\omega_{\mathrm{eg}}^{*}(\tau)$ and $\alpha(\tau)$, that will be extracted from experimental data. The window function may then be written as

$$
\begin{aligned}
W_{l u v}\left(\tau, \omega_{\mathrm{det}}\right)= & \frac{\langle v \mid u\rangle\langle v \mid l\rangle}{\sqrt{4 \pi \lambda k_{\mathrm{B}} T}} \exp \left\{-\frac{\left[\omega_{\mathrm{det}}-\omega_{\mathrm{eg}}^{*}(\tau)\right]^{2}}{2 \alpha^{2}(\tau)}\right\} \\
& \times \cos \left(\omega_{u} \tau\right) \exp \left(-\Gamma_{u} \tau\right)
\end{aligned}
$$

where $\omega_{\operatorname{det}}$ is the detection frequency in the transient absorption experiment. Most generally, the transient absorption signal associated with the signal component of interest is then obtained by summing over the product of $D_{n k \rightarrow u l}(\tau)$ and $W_{l u v}\left(\tau, \omega_{\mathrm{det}}\right)$,

$$
S\left(\tau, \omega_{\mathrm{det}}\right)=\sum_{m n k l u v} D_{n k \rightarrow u l}(\tau) W_{l u v}\left(\tau, \omega_{\mathrm{det}}\right)
$$

Equation 18 incorporates all four pathways in Figure 1. The summation can be restricted to select incoherent and coherent components.

IIC. Spectral Fitting of Absorbance and Resonance Raman Cross Sections. Iterative fitting of both the linear absorbance spectrum and the resonance Raman cross sections imposes constraints on the displacement of intramolecular modes, the electronic energy gap, the solvent reorganization energy, and the relative contributions of homogeneous and inhomogeneous line broadening mechanisms. ${ }^{37,39}$ Many of these same parameters also control nonradiative BET in systems that possess charge-transfer resonances. ${ }^{28}$ We summarize essential aspects of the model in this section. Additional background on the approach can be found elsewhere. ${ }^{39,40}$

In a basis set of delocalized excited states, $\left[\mathrm{Ti}(\mathrm{cat})_{3}\right]^{2-}$ possesses two allowed LMCT transitions and one (higher energy) forbidden LMCT transition; these transitions correspond to the $\mathrm{E}$ and $\mathrm{A}$ irreducible representations of the $C_{3}$ point group, respectively. This aspect of the electronic structure is taken into account in the expressions for the absorption and resonance Raman cross sections given in this section. The best choice of a basis set in a transition metal complex is not necessarily obvious and can even be dynamic in some cases. $^{41-44}$ Energy level fluctuations induced by solute-solvent interactions tend to localize the excited states in transition metal complexes, whereas electronic coupling between ligands promotes delocalization. ${ }^{41-43}$ Charge-transfer states should be treated as localized if the interligand electronic couplings are much smaller than the energy level fluctuations. Such general rules of thumb have been applied extensively to distinguish localized electronic excitations from Frenkel excitons in molecular aggregates, molecular crystals, and photosynthetic complexes. $^{16,45-48}$ Frenkel exciton-like models have been adapted to transition metal complexes in recent work. ${ }^{49,50}$ We opt for a delocalized basis set in $\left[\mathrm{Ti}(\mathrm{cat})_{3}\right]^{2-}$, because estimates of the local (interligand) electronic couplings obtained with density functional theory are consistent with an intermediate regime, where valid arguments can be made for both basis sets (Supporting Information). In the intermediate regime, the extent of delocalization is distributed in the ensemble. ${ }^{43}$ We emphasize that the main conclusions drawn in this work are independent of the basis set of the system.

The linear absorbance line shape of $\left[\mathrm{Ti}(\mathrm{cat})_{3}\right]^{2-}$ is given by

$$
\begin{aligned}
\sigma_{\mathrm{A}}(\omega)= & \frac{8 \pi \omega \mu_{\mathrm{eg}}{ }^{2}}{3 \hbar c} \sum_{m=1} B_{m} \operatorname{Re} \int_{-\infty}^{\infty} \mathrm{d} \delta G(\delta) \int_{0}^{\infty} \mathrm{d} t\langle m \mid m(t)\rangle \\
& \times \exp \left[\mathrm{i}\left(\omega-\omega_{0}-\delta+\omega_{m}\right) t-\gamma(t)\right]
\end{aligned}
$$

where $m$ is an index for a nuclear mode, $B_{m}$ is the Boltzmann population of the initial state, $\mu_{\mathrm{eg}}$ is the electronic transition dipole, $\omega_{m}$ is the frequency associated with vibrational state $m$, $\omega_{0}$ is the purely electronic transition frequency (i.e., electronic origin), and $\langle m \mid m(t)\rangle$ is a dynamic overlap integral between nuclear wave functions (the Fourier transform of $\langle m \mid m(t)\rangle$ is a Franck-Condon progression). The dynamic overlap integral, $\langle m \mid m(t)\rangle$, is controlled by the mode frequencies and dimensionless potential energy surface displacements. Fluctuations in the electronic resonance frequency induced by thermal motion in the solvent are partitioned into fast (homogeneous) and slow (inhomogeneous) components. Inhomogeneous line broadening is incorporated by integrating over the static Gaussian distribution, $G(\delta)$, whereas the homogeneous line shape is captured by the line broadening function, $\gamma(t)$ (eq 4 ).

The differential resonance Raman cross section is written as 


$$
\begin{aligned}
\sigma_{R, m l}(\omega)= & \frac{8 \omega_{\mathrm{S}}^{3} \omega_{\mathrm{L}}}{15 c^{4}} \sum_{m l} B_{m} \int \mathrm{d} \omega_{\mathrm{S}} \int_{-\infty}^{\infty} \mathrm{d} \delta G(\delta) \\
& \times L_{m l}\left(\omega_{\mathrm{L}}-\omega_{\mathrm{S}}\right)\left|\alpha_{m l}\left(\omega_{\mathrm{L}}, \delta\right)\right|^{2}
\end{aligned}
$$

where $m(l)$ is an index for the initial (final) vibrational state, $\omega_{\mathrm{L}}\left(\omega_{\mathrm{S}}\right)$ is the incident (scattered) light frequency, and $L_{m l}\left(\omega_{\mathrm{L}}\right.$ $-\omega_{S}$ ) is the normalized line shape of the $m \rightarrow l$ ground-state vibrational transition. The integral with respect to the scattered frequency, $\omega_{\mathrm{S}}$, extends over the full Raman emission band. The Raman polarizability of $\left[\mathrm{Ti}(\mathrm{cat})_{3}\right]^{2-}$ is given by

$$
\begin{aligned}
\alpha_{m l}\left(\omega_{L}, \delta\right)= & \frac{2 \mu_{\mathrm{eg}}{ }^{2}}{\hbar} \int_{0}^{\infty} \mathrm{d} t\langle\operatorname{llm}(t)\rangle \\
& \times \exp \left[\mathrm{i}\left(\omega_{\mathrm{L}}-\omega_{0}-\delta+\omega_{m}\right) t-\gamma(t)\right]
\end{aligned}
$$

Dependence of the optical response and electron-transfer rate on the same parameters has been leveraged for physical insight in earlier work. ${ }^{6,27-29}$ The short-time approximation was taken in section IIB to obtain Gaussian functions, whereas the more general form of $\gamma(t)$ appears in $\sigma_{\mathrm{A}}(\omega)$ and $\alpha_{m l}\left(\omega_{\mathrm{L}} \delta\right)$. The ratio $\Lambda / \Delta$ is generally much less than 1 for electronic transitions in polar solvents at room temperature; ${ }^{51,52}$ that is, significant motional narrowing is generally not observed judging by Gaussian line shapes and Stokes shifts. The classical reorganization energy associated with electron transfer, $\lambda=D^{2}$ / $2 k_{\mathrm{B}} T$, can be computed with

$$
D=\Gamma\left[\frac{1+0.85 \kappa+0.88 \kappa^{2}}{2.355+1.76 \kappa}\right]
$$

where the homogeneous line width is given by $\Gamma=\lambda k_{\mathrm{B}} T / \hbar \Lambda$. We note that $D \approx \Delta$ when $\Lambda / \Delta \ll 1$ (eq 5).

IID. Summary of Assumptions and Parameters. In this section, we summarize key assumptions associated with the models for transient absorption and resonance Raman experiments. Some standard approximations that are inherent to the correlation function approach are discussed in detail elsewhere. ${ }^{14-16}$ Briefly, it is assumed in all cases that energy level fluctuations induced by the bath possess Gaussian statistics. We further assume that the thermal fluctuation amplitudes are large compared to the inverse of the relaxation time of the bath (i.e., the slow modulation limit). In this limit, we have shown that a closely related model can be reduced to the traditional Marcus equation at times that are long compared to solvation. ${ }^{31}$

The nine key assumptions made in sections IIA, IIB, and IIC are

(i) Perturbative interactions with the donor-acceptor coupling, $V_{\text {eg }}$ and pump laser pulse, $\vec{E} \cdot \vec{\mu}_{\mathrm{eg}}$, have been assumed. The low pulse energy of the pump laser justifies this assumption. That is, the experiments are conducted in a regime where the dynamics are insensitive to the pulse energy. Explicit forms for the donor-acceptor coupling and transition dipole operators are not proposed, because they enter the model as prefactors that do not affect the spectroscopic line shapes under the Condon approximation.

(ii) It has been assumed that two perturbative interactions with the pump laser pulse occur before the two perturbative interactions with the donor-acceptor coupling (Figure 2). This assumption is justified by the large energy gap $\left(20100 \mathrm{~cm}^{-1}\right)$ between the ground and excited states (i.e., spontaneous electron transfer is negligible in the equilibrium system).

(iii) The solvent coordinate is taken to be overdamped. This assumption is justified by the broad Gaussian-like absorbance line shape.

(iv) The 17 fs pump laser pulse is assumed to be short compared to the $180 \mathrm{fs}$ time scale of BET.

(v) The BET process is taken to be fast compared to transitions between excited states.

(vi) The laser spectrum is assumed to be narrower than the absorbance line width.

(vii) In section IIB, vibronic structure in $t_{1}$ is neglected on the basis of the absence of a Franck-Condon progression in the absorbance spectrum.

(viii) A basis of "delocalized" electronic states is assumed in section IIC. This basis set is justified by the similar magnitudes of the energy level fluctuations and the interligand coupling, which has also been referred to as the coupling associated with "dipole reorientation". ${ }^{53}$ This point is further discussed in the Supporting Information. Conclusions drawn in this work are independent of the basis set.

(ix) Modes that are Franck-Condon active in light absorption are also Franck-Condon active in the BET process, because $\left[\mathrm{Ti}(\mathrm{cat})_{3}\right]^{2-}$ possesses a charge-transfer resonance. Numerous earlier works have leveraged this aspect of systems with charge-transfer bands in studies of electron-transfer mechanisms. ${ }^{6,27-29}$

The models used to simulate the transient absorption signals and the resonance Raman cross sections are based on the same parameters. The parameters of the models are

(i) The free energy gap between the ground and excited state is referred to as $\Delta G_{\mathrm{eg}}^{0}$ in the model for transient absorption in section IIB. ${ }^{1,31}$ This quantity corresponds to the optical $0-0$ frequency in the resonance Raman model $\left(\omega_{0}\right.$ in section IIC) ${ }^{28}$

(ii) The solvent reorganization energy is denoted as $\lambda$. This quantity is derived from the homogeneous component of the line shape determined by the resonance Raman intensity analysis, $\lambda=D^{2} / 2 k_{\mathrm{B}} T$ (see eq 22 ).

(iii) The normal mode displacements used to evaluate vibrational overlap integrals are determined with the resonance Raman intensity analysis.

(iv) The transition dipole magnitude is determined by the resonance Raman intensity analysis with constraints imposed by earlier work. ${ }^{23}$

(v) Knowledge of the donor-acceptor coupling, $V_{\text {eg }}$ and electric field magnitude, $\xi_{\mathrm{L}}$, are not required to compute the transient absorption response, because they enter eq 14 as a constant prefactor.

(vi) The frequency, $\omega_{\mathrm{L}}$, and width, $w_{\mathrm{L}}$, of the pump laser pulse are determined by measuring the laser spectrum.

\section{EXPERIMENTAL METHODS}

IIIA. Sample Preparation. Synthesis of $\left[\mathrm{Ti}(\mathrm{cat})_{3}\right]^{2-}$ is carried out using the procedure described in ref 23 . The aqueous solutions are flowed through a wire-guided jet with a thickness of $300 \mu \mathrm{m}$, where the volume of the reservoir is 50 $\mathrm{mL}$. The absorbance of the solution is equal to 0.5 at $408 \mathrm{~nm}$ in $300 \mu \mathrm{m}$ path length. Absorbance spectra are acquired before and after experiments to confirm the absence of sample degradation. 
IIIB. Raman Spectroscopy. Resonance Raman spectra are collected with a Renishaw Via- Leica DM2500 M microscope. Excitation is accomplished using the 458, 488, and $515 \mathrm{~nm}$ laser lines of an argon ion laser (Spectra Physics Stabilite 2017). The $2 \mathrm{~mW}$ laser beams are focused into $1 \mathrm{~mm}$ thick cuvettes with a Leica N PLAN EPI 20x/0.4 objective lens, which is also used to collect the scattered Raman emission in a confocal geometry. A polarization scrambler is placed between the sample and detector during measurements. ${ }^{39}$ Reabsorption of the Raman emission is corrected using an established procedure. ${ }^{54}$ The signals are averaged for 10-20 min with integration times of 30 s. Cosmic spikes are removed using software provided by Renishaw. The solutions contain a mixture of $\left[\mathrm{Ti}(\mathrm{cat})_{3}\right]^{2-}(6$ $\mathrm{mmol} / \mathrm{L})$ and sulfate $(850 \mathrm{mmol} / \mathrm{L})$, which is used as an internal standard. ${ }^{55}$ All experiments are repeated three times with fresh solutions to establish reproducibility of the Raman cross sections.

IIIC. Transient Absorption Experiments. Transient absorption experiments are carried out with a $1 \mathrm{kHz}, 1 \mathrm{~mJ}$ Ti:sapphire laser system. Pump pulses are produced by doubling the frequency of the fundamental laser beam in a $0.25 \mathrm{~mm}$ thick BBO crystal. The resulting $90 \mu \mathrm{J}, 408 \mathrm{~nm}$ second harmonic beam is then focused with a $30 \mathrm{~cm}$ focal length lens into a $75 \mathrm{~cm}$ long, $100 \mu \mathrm{m}$ inner diameter hollow core fiber. The fiber is housed in a stainless steel cell filled with $1.0 \mathrm{~atm}$ of argon gas. Compression of the pump pulse to $17 \mathrm{fs}$ (fwhm of intensity) is achieved with a fused silica prism compressor with a $50 \mathrm{~cm}$ prism separation. Probe laser pulses are generated using either $\mathrm{CaF}_{2}$ or sapphire windows. The $\mathrm{CaF}_{2}$ window is used to acquire signals from 350 to $700 \mathrm{~nm}$ with the magic angle polarization condition. Isotropic and anisotropic vibrational motions are detected with probe pulses generated in sapphire. Slightly better signal-to-noise (a factor of 5-10 improvement) is achieved with sapphire because the window is stationary, whereas the $\mathrm{CaF}_{2}$ window must be continuously moved to avoid damage. The probe pulses are directed to the sample with reflective optics and do not pass through the entrance window of a cuvette.

The $408 \mathrm{~nm}$ pump pulsed is focused onto the sample jet with a $30 \mathrm{~cm}$ focal length spherical mirror, whereas the continuum is relayed from the sapphire plate onto the sample using a single 5 $\mathrm{cm}$ focal length mirror (the continuum focuses $35 \mathrm{~cm}$ from the spherical mirror). The fwhm spot sizes of the $408 \mathrm{~nm}$ pump beam is $600 \mu \mathrm{m}$, whereas that of the continuum is $400 \mu \mathrm{m}$. The pulse energy of the pump is $200 \mathrm{~nJ}$ at the sample position. The angle between the two beams is $5^{\circ}$. Signal detection is accomplished with a CMOS array detector that is synchronized to the $1 \mathrm{kHz}$ repetition rate of the laser system. The pump beam passes through a chopper wheel operated at $500 \mathrm{~Hz}$, thereby enabling the acquisition of absorption spectra with and without the pump pulse on a shot-to-shot basis. The signals are averaged over 30-40 scans of the delay line if the magic angle polarization condition is employed. Isotropic and anisotropic vibrational motions are compared by interleaving 100 scans of the delay line with parallel and perpendicular pump and probe polarizations. The interleaving of scans is achieved by mounting a half-waveplate in a motorized rotation stage (the pump beam passes through the waveplate). In all cases, a calcite polarizer is located between the sample and detector to ensure that the polarization condition is well-defined.

\section{RESULTS AND DISCUSSION}

In this section, resonance Raman cross sections are used to determine the electronic energy gap, reorganization energy, and vibrational mode displacements of $\left[\mathrm{Ti}(\mathrm{cat})_{3}\right]^{2-}$. Transient absorption experiments are then used to detect vibrational coherences associated with the two pathways that end in the upper right of Figure 1. Finally, the BET mechanism is simulated using empirical parameters determined by fitting the resonance Raman and transient absorption data.

IVA. Resonance Raman Intensity Analysis and Spectral Fitting. In Figure 3, the low-energy side of the

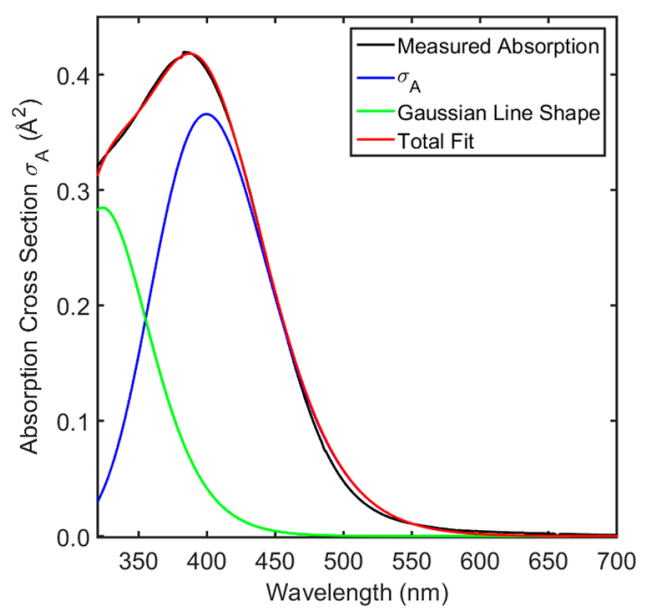

Figure 3. Measured absorption spectrum fit with eq 19 and the parameters in Table 1 . The absorption cross section, $\sigma_{A}$, is used to fit the low-energy side of the line shape subject to the constraints imposed by the Raman cross sections. An additional Gaussian line shape (green) is used to estimate the contribution of the second-tolowest energy transition to the total absorbance.

absorption spectrum is fit using eq 19 and the parameters in Table 1 . The rise of the band at longer wavelengths imposes useful constraints, because the line shape is quite sensitive to the relative contributions of the intramolecular and solvent reorganization energies. Inflation of the homogeneous width increases the Lorentzian character of the line shape, whereas the inhomogeneous contribution is purely Gaussian. An additional Gaussian line shape is added to the fit at shorter wavelengths to estimate the contribution of the second-tolowest energy electronic transition to the total absorbance spectrum.

The resonance Raman spectrum of $\left[\mathrm{Ti}(\mathrm{cat})_{3}\right]^{2-}$ acquired with excitation at $488 \mathrm{~nm}$ is shown in Figure 4. In Table 1, we suggest tentative assignments of the normal modes using a calculation carried out at the B3LYP/6-311G(2d,3p) level of theory in Gaussian 2009. ${ }^{56}$ The assignments are based on agreement between the experimental and calculated vibrational frequencies in addition to aspects of the nuclear motion. As in earlier work, ${ }^{51}$ we assume that the most intense transitions involve modes with larger reduced masses (i.e., coordinates of the hydrogen atoms are weakly coupled to charge transfer). It is also assumed that the response is dominated by in-plane motions of the catechol ligands, which preserve bond conjugation in the aromatic rings. For most of these assignments, the motion described in Table 1 applies to a number of "candidate modes" with similar frequencies. Thus, most of the descriptions (e.g., Ti-cat stretch, $\mathrm{C}=\mathrm{C}$ stretch) are 
Table 1. Resonance Raman Fitting Parameters

\begin{tabular}{|c|c|c|}
\hline \multicolumn{2}{|c|}{ parameter } & value \\
\hline \multicolumn{2}{|l|}{$\omega_{0}^{a}$} & $20100 \pm 3000 \mathrm{~cm}^{-1}(476 \pm 68 \mathrm{~nm})$ \\
\hline \multicolumn{2}{|l|}{$\delta^{a}$} & $2100 \pm 500 \mathrm{~cm}^{-1}(52 \pm 12 \mathrm{~nm})$ \\
\hline \multicolumn{2}{|c|}{$\Gamma^{a}$} & $2700 \pm 660 \mathrm{~cm}^{-1}(67 \pm 16 \mathrm{~nm})$ \\
\hline \multicolumn{2}{|c|}{$\kappa=\Delta / \Lambda$} & 0.01 \\
\hline \multicolumn{2}{|c|}{$\lambda^{b}$} & $3290 \pm 785 \mathrm{~cm}^{-1}$ \\
\hline \multicolumn{2}{|l|}{$\mu_{\mathrm{eg}}$} & $4.1 \pm 0.4 \mathrm{D}$ \\
\hline$\underset{\left(\mathrm{cm}^{-1}\right)}{\text { Raman shift }}$ & $\begin{array}{l}\text { dimensionless } \\
\text { displacement }\end{array}$ & assignment $^{c}$ \\
\hline 222 & $1.24 \pm 0.37$ & symmetric Ti-cat stretch $\left(214 \mathrm{~cm}^{-1}\right)$ \\
\hline 410 & $0.71 \pm 0.21$ & Ti-cat bend $\left(413 \mathrm{~cm}^{-1}\right)$ \\
\hline 538 & $0.50 \pm 0.15$ & $\begin{array}{l}\mathrm{O}-\mathrm{C}-\mathrm{C}-\mathrm{O} \text { bend and ring distortion } \\
\left(534 \mathrm{~cm}^{-1}\right)\end{array}$ \\
\hline 643 & $1.11 \pm 0.33$ & $\begin{array}{l}\mathrm{O}-\mathrm{C}-\mathrm{C}-\mathrm{O} \text { bend and ring distortion } \\
\left(641 \mathrm{~cm}^{-1}\right)\end{array}$ \\
\hline 811 & $0.47 \pm 0.14$ & $\begin{array}{l}\mathrm{O}-\mathrm{C}-\mathrm{C}-\mathrm{O} \text { bend and ring "breathing" } \\
\left(814 \mathrm{~cm}^{-1}\right)\end{array}$ \\
\hline 1153 & $0.20 \pm 0.06$ & $\mathrm{C}-\mathrm{H}$ bending $\left(1156 \mathrm{~cm}^{-1}\right)$ \\
\hline 1262 & $0.53 \pm 0.16$ & $\mathrm{O}-\mathrm{C}-\mathrm{C}-\mathrm{O}$ stretch $\left(1283 \mathrm{~cm}^{-1}\right)$ \\
\hline 1330 & $0.53 \pm 0.16$ & $\begin{array}{l}\mathrm{C}=\mathrm{C} \text { stretch, } \mathrm{C}-\mathrm{O} \text { stretch, \& } \mathrm{Ti}-\mathrm{O} \\
\text { stretch }\left(1305 \mathrm{~cm}^{-1}\right)\end{array}$ \\
\hline 1482 & $0.68 \pm 0.20$ & $\mathrm{C}=\mathrm{C}$ stretch \& $\mathrm{C}-\mathrm{O}$ stretch $\left(1511 \mathrm{~cm}^{-1}\right)$ \\
\hline 1580 & $0.19 \pm 0.06$ & $\mathrm{C}=\mathrm{C}$ stretch $\left(1594 \mathrm{~cm}^{-1}\right)$ \\
\hline
\end{tabular}

${ }^{a}$ Fits are carried out in the frequency domain. ${ }^{b}$ Computed with $\lambda=$ $D^{2} / 2 k_{\mathrm{B}} T$, where $D$ is defined with eq $22 .{ }^{c}$ Assignments for vibrational modes computed at the B3LYP/6-311G $(2 \mathrm{~d}, 3 \mathrm{p})$ level in Gaussian $2009 .{ }^{56}$ Frequencies of the calculated modes are given in parentheses.

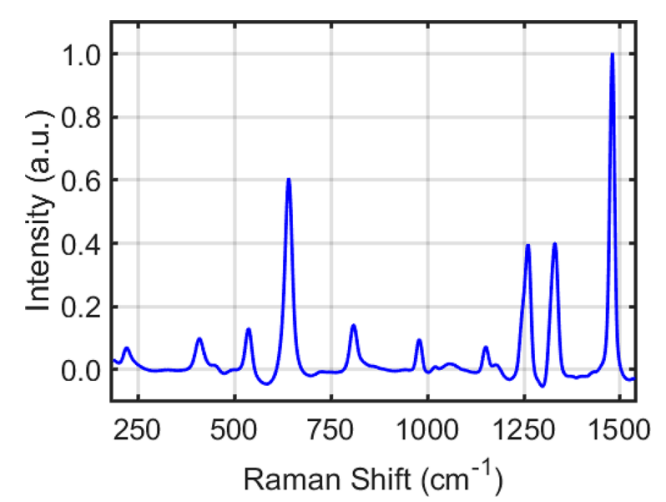

Figure 4. Resonance Raman spectrum of $\left[\mathrm{Ti}(\mathrm{cat})_{3}\right]^{2-}$ acquired in aqueous solution with excitation at $488 \mathrm{~nm}$.

likely to hold even if assignments to particular modes are later revised.

A full resonance Raman excitation profile cannot be generated because of available laser sources and contributions from higher-energy electronic resonances to the charge-transfer band at shorter wavelengths. Nonetheless, determination of the Raman cross sections on the lower-energy side of the chargetransfer resonance still imposes strong constraints on the line broadening parameters, because the resonance Raman cross sections increase as the inhomogeneous component of the absorbance line shape increases. Experimental and calculated Raman cross sections are compared in Figure 5.

Simultaneous fitting of absorbance and resonance Raman cross sections has been carried out in numerous earlier works. $^{28,39,51,52}$ It is not straightforward to produce error bars for this highly nonlinear model. ${ }^{57}$ Therefore, we have explored fits in different regions of parameter space. For example, a decrease in the homogeneous line width is generally
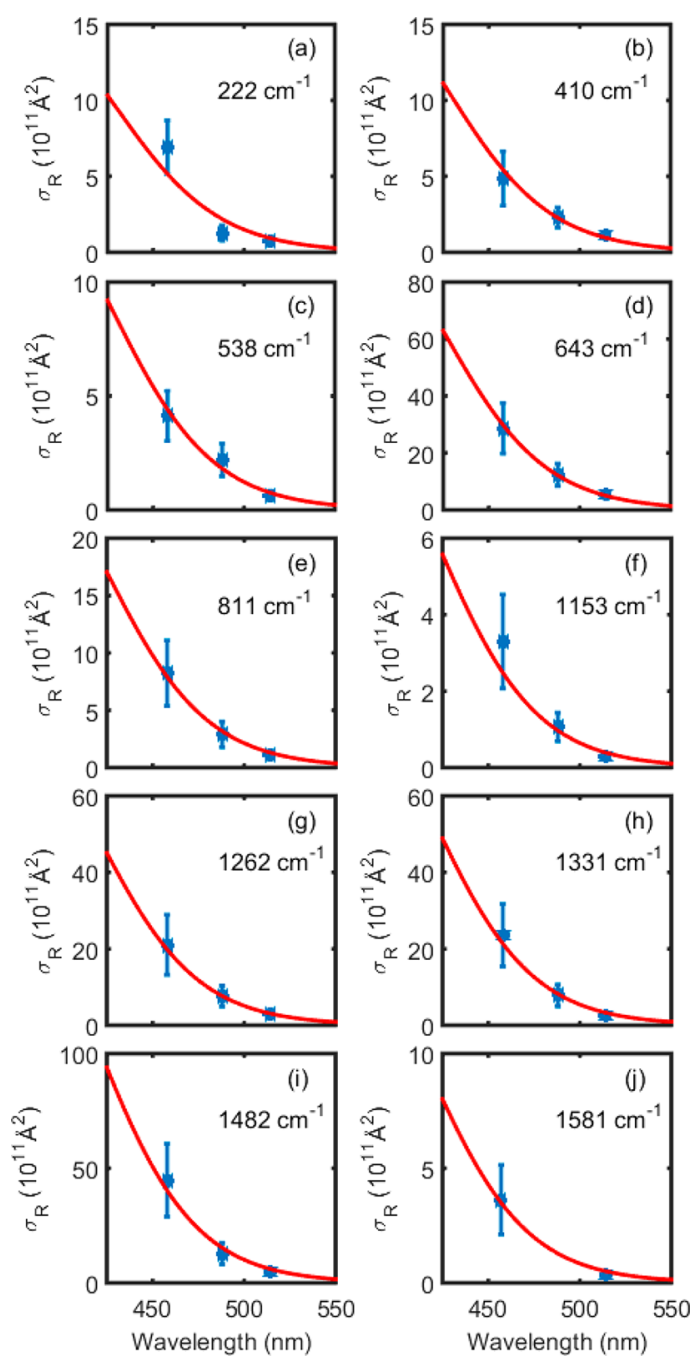

Figure 5. Experimental Raman cross sections are fit using eq 20 and the parameters in Table 1 .

compensated for by increasing the electronic origin, increasing the inhomogeneous width, and decreasing the mode displacements. We find that the line widths and mode displacements can be changed by roughly $25 \%$ and $33 \%$ before large discrepancies between the measurements and fits are produced. The error bars in Table 1 reflect these percentages.

The model yields the best fit for homogeneous and inhomogeneous line widths of 2700 and $2100 \mathrm{~cm}^{-1}$, respectively. The homogeneous line width can be converted into a classical solvent reorganization energy of $3290 \mathrm{~cm}^{-1}$ using the Padé approximant. ${ }^{14,51,52}$ This is perhaps a modest amount of reorganization energy for an electron-transfer transition in aqueous solution. However, it was noted in earlier work that the Padé approximant may underestimate solvent reorganization energies in hydrogen bonding solvents. ${ }^{51,52}$ In such cases, the inhomogeneous line width may account for a portion of the "slower" solvent reorganization. The dominant low-frequency modes at 222 and $643 \mathrm{~cm}^{-1}$ involve motions on the linkers between titanium and the ligands. Intense transitions above $1000 \mathrm{~cm}^{-1}$ mostly correspond to bondstretching motions on the aromatic rings. The higher-frequency modes with large displacements (at 1262, 1330, and 1482 $\mathrm{cm}^{-1}$ ) carry important implications for BET, because they 

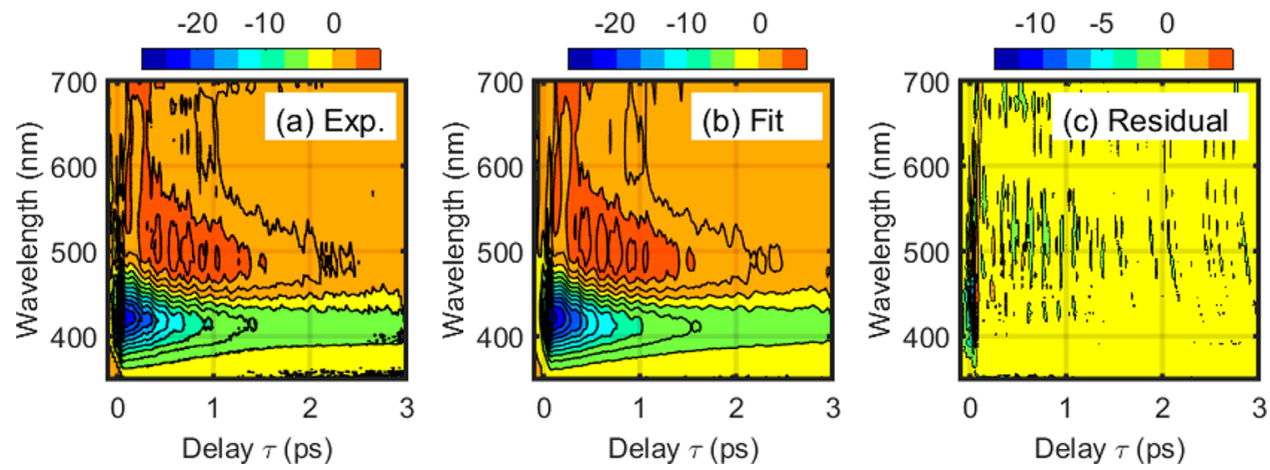

Figure 6. (a) Experimental transient absorption signal acquired under the magic angle polarization condition is (b) fit using eq 23 . The residual shows that agreement between the experiment in fit is best at delay times greater than $60 \mathrm{fs}$ (i.e., outside the region of pulse overlap).
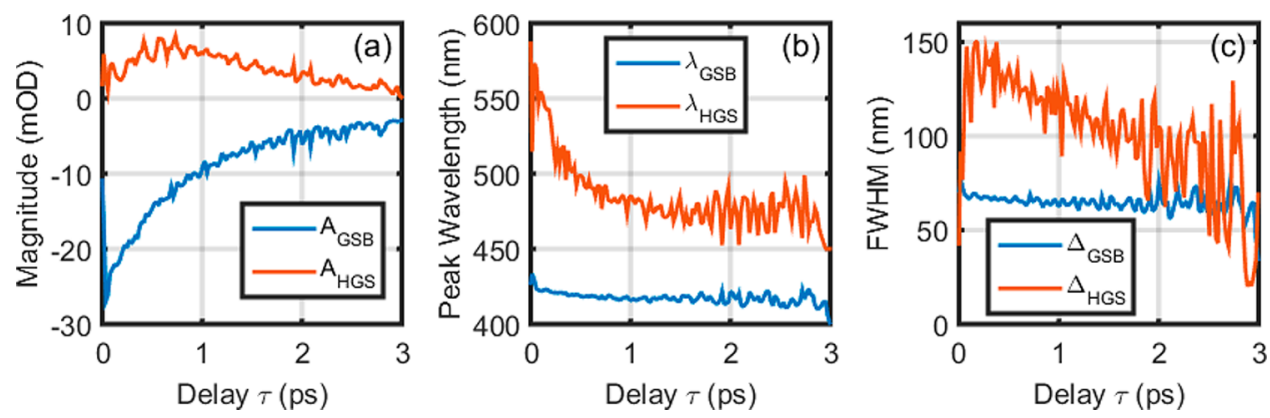

Figure 7. (a) The magnitude of the HGS signal component rises until a delay time of 0.5 ps before vibrational cooling causes the signal to decay. The GSB signal component rises instantaneously and decays on the time scale of vibrational cooling. (b) The HGS signal component shifts to shorter wavelengths because of vibrational cooling, whereas the peak of the GSB resonance is insensitive to the delay time. (c) The fwhm of the HGS resonance decreases by $50 \%$ within the first 3 ps, whereas the line width of the GSB is insensitive to the delay time. The 100 fs time scale of the BET process is the key information provided by these data.

possess the largest vibrational wave function overlaps with the ground vibrational level of the excited electronic state.

IVB. Decomposition of Transient Absorption Signal Components. Photoexcitation of the charge-transfer resonance at $408 \mathrm{~nm}$ induces electron transfer from the catechol ligands to the titanium atom. In Figure 6, the decrease in absorbance near $420 \mathrm{~nm}$ represents depopulation of the ground electronic state. This nonlinearity is denoted as the groundstate bleach (GSB). BET from titanium to catechol is evidenced by growth of an absorptive response near $520 \mathrm{~nm}$ on the $100 \mathrm{fs}$ time scale. ${ }^{24}$ This signal component is referred to as the "hot ground state" (HGS). The HGS resonance is observed at longer wavelengths, because BET leaves the molecule in a highly nonequilibrium state that subsequently relaxes through vibrational energy transfer to the solvent. The HGS response blue-shifts with increasing delay because of vibrational energy transfer to the solvent. Molecules that relax to the ground electronic state on the subpicosecond time scale generally exhibit a blue-shifting HGS nonlinearity. ${ }^{58,59}$ This effect becomes more pronounced as the resonance frequency with the lowest energy excited state increases because of a concomitant increase in the amount of heat that is dissipated following ground-state recovery.

In addition to the GSB and HGS signal components, the transient absorption signal exhibits a broadband feature in the near-infrared (denoted with the BB subscript below). ${ }^{24}$ These three resonances are fit to establish their relative contributions in the present measurements, which are carried out with dispersed detection on a CMOS array (i.e., some improvement in signal-to-noise is achieved compared to earlier work based on single channel detection). ${ }^{24}$ These three signal components are fit with the following function,

$$
\begin{aligned}
F(\tau, \lambda)= & A_{\mathrm{GSB}}(\tau) \exp \left\{-4 \ln (2) \frac{\left[\lambda-\lambda_{\mathrm{GSB}}(\tau)\right]^{2}}{\Delta_{\mathrm{GSB}}^{2}(\tau)}\right\} \\
& +A_{\mathrm{HGS}}(\tau) \exp \left\{-4 \ln (2) \frac{\left[\lambda-\lambda_{\mathrm{HGS}}(\tau)\right]^{2}}{\Delta_{\mathrm{HGS}}^{2}(\tau)}\right\} \\
& +A_{\mathrm{BB}}(\tau) \exp \left\{-4 \ln (2) \frac{\left[\lambda-\lambda_{\mathrm{BB}}(\tau)\right]^{2}}{\Delta_{\mathrm{BB}}^{2}(\tau)}\right\}
\end{aligned}
$$

We do not include excited-state emission or excited-state absorption resonances for two reasons: (i) previous work on this system has shown that the upper limit of the excited-state lifetime is $180 \pm 50 \mathrm{fs}$, ${ }^{24}$ whereas the vibrational coherences of interest in this study dephase in 1-2 ps; (ii) the fit in Figure 6 is quite good outside of the region of pulse overlap, which supports the physical picture associated with eq 23. Constraints must be imposed for such a multivariable fit to converge; however, the ranges applied to each parameter are "loose" and physically reasonable. The lower and upper limits for $\lambda_{\mathrm{GSB}}(\tau)$ are 375 and $440 \mathrm{~nm}$. The lower and upper limits for $\lambda_{\mathrm{HGS}}(\tau)$ are 450 and $620 \mathrm{~nm}$, respectively. For both $\Delta_{\mathrm{GSB}}(\tau)$ and $\Delta_{\mathrm{HGS}}(\tau)$, the lower and upper limits are 15 and $150 \mathrm{~nm}$. The values of $A_{\mathrm{HGS}}(\tau)$ and $A_{\mathrm{GSB}}(\tau)$ range from 0 to +30 and -50 $\mathrm{mOD}$, respectively.

Parameters associated with the GSB and HGS resonances, which are displayed in Figure 7, are of primary interest. The GSB response rises instantaneously and subsequently decays because of vibrational energy transfer to the solvent. In 
contrast, the magnitude of the HGS response rises for $500 \mathrm{fs}$ before vibrational cooling induces decay. Large-amplitude oscillations in the HGS signal magnitude are observed at a wavenumber of $222 \mathrm{~cm}^{-1}$ in the first picosecond; however, vibrational coherences are not detected in the GSB. The peak of the HGS response blue-shifts as vibrational energy is transferred into the surrounding solvent, whereas the peak of the GSB response is essentially delay-independent. The line width of the HGS also decreases on the picosecond time scale, whereas the line width of the GSB is insensitive to the delay time. Transient blue-shifting and line-narrowing in the HGS signal component were observed in previous work on $\left[\mathrm{Ti}(\mathrm{cat})_{3}\right]^{2-24}$ These behaviors of the HGS signal component are indicative of vibrational cooling following ultrafast groundstate recovery. Systems such as nucleobases and $p$-nitroaniline exhibit similar spectroscopic signatures. ${ }^{58,59}$

A broadband signal component is included in the fit at both short and long delay times, because a residual response in excess of $50 \%$ of the total signal strength is found without this term in eq 23. The resonance frequency of the broadband signal component is apparently red-shifted beyond the limit of our detection range at $700 \mathrm{~nm}$. The picosecond rate of decay suggests that this resonance relaxes on the time scale of vibrational cooling; however, relatively weak transient absorption persists in the near-infrared even at delay times of hundreds of picoseconds. Lian and co-workers, who conducted earlier experiments on $\left[\mathrm{Ti}(\mathrm{cat})_{3}\right]^{2-}$, suggested that one of the bonds between catechol and the titanium atom may detach following photoexcitation then reattach after hundreds of picoseconds. $^{24}$ We hypothesize that this species is also responsible for the broadband, red-shifted signal component in our experiments. Because the response appears to be redshifted beyond our detection range, we have constrained both $\lambda_{\mathrm{BB}}(\tau)$ and $\Delta_{\mathrm{BB}}(\tau)\left(650 \mathrm{~nm}<\lambda_{\mathrm{BB}}(\tau)<850\right.$ and $400 \mathrm{~nm}<$ $\left.\Delta_{\mathrm{BB}}(\tau)<1000 \mathrm{~nm}\right)$. These constraints were required to eliminate discontinuities in the fit.

IVC. Initiation of Vibrational Coherence by BackElectron-Transfer. The pathways of interest in this work terminate in the upper right of Figure 1. In these pathways, both vibrational populations and coherences are first initiated in the excited electronic state. The BET process then induces vibrational coherences in the ground electronic state. Vibrational oscillations in the HGS signal component are clear evidence of contributions from the two coherent pathways in Figure 1, because light absorption and BET have the same Franck-Condon active modes in systems that possess a chargetransfer band. ${ }^{6,27-29}$ The key issue is that the gradient of the potential energy must change when the BET process occurs, because the potential energy minima are displaced for FranckCondon active modes. For systems that do not exhibit a chargetransfer band, it would be possible for "spectator modes", whose potential energy minima are not displaced in both optical and electron-transfer transitions, to contribute in transient absorption experiments; this would be undesirable in the present study, because spectator modes are largely irrelevant to electron-transfer mechanisms.

Transient absorption signals are Fourier transformed in Figure 8 to reveal the dependence of vibrational motion on the detection wavelength. The relatively weak oscillations exhibited by the GSB nonlinearity in the $350-450 \mathrm{~nm}$ wavelength range underscores the distinction between BET-induced (HGS) and radiation-induced (GSB) vibrational coherences. ${ }^{60}$ The stimulated Raman response in the GSB nonlinearity is highly

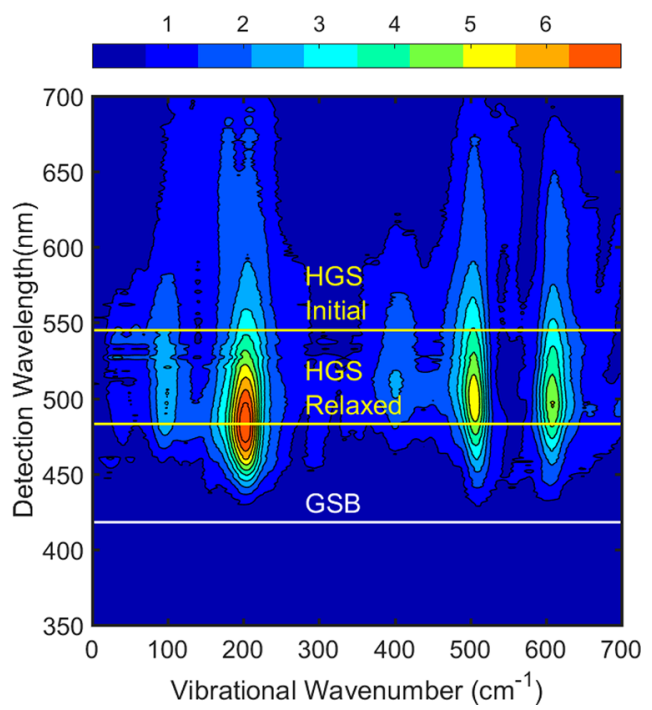

Figure 8. Coherent component of the transient absorption response of $\left[\mathrm{Ti}(\mathrm{Cat})_{3}\right]^{2-}$ analyzed for a range of detection wavelengths. Coherent vibrational motion is detected at wavelengths that correspond to the HGS signal component. The stimulated Raman response for the GSB signal component is comparable to the noise level of the experiment. This measurement demonstrates that the BET process initiates vibrational motions, which represent pathways that end in the upper right of Figure 1.

dependent on the electronic dephasing time. That is, the GSB wavepacket accumulates force in the interval between the first two field-matter interactions, $t_{1}$ (Figure 2). ${ }^{14}$ A short electronic dephasing time of 12 fs (inverse of homogeneous line width) ensures that the GSB wavepacket is initiated with a small displacement from the equilibrium geometry. Such dependence on electronic dephasing is one reason why lower-frequency modes generally have smaller resonance Raman cross sections than a higher-frequency mode with an identical displacement. In contrast, the BET-induced wavepacket detected in the 450$550 \mathrm{~nm}$ wavelength range accumulates force when it evolves on the excited-state potential energy surface during a population time, $t_{2}$ in Figure 2, and is therefore less sensitive to electronic dephasing (i.e., the wavepacket evolves on the excited-state potential during the $180 \mathrm{fs}$ excited-state lifetime). The BET process deposits the wavepacket on the ground-state potential in a location that may be relatively far-displaced from the Franck-Condon geometry. In Figure 8, the BET-induced wavepacket generates the largest oscillations in the transient absorption signals, because its motion is induced by large forces on the ground-state potential.

The transient absorption signals in Figure 9 show that vibrational coherences are quite prominent in the HGS signal component; the recurrences reach $25 \%$ of the total signal magnitude in the $450-550 \mathrm{~nm}$ range. Isotropic and anisotropic signals are analyzed near the peak of the signal strength at 500 $\mathrm{nm}$ to facilitate a better understanding of the HGS response. The isotropic signal component is produced by combining signals measured with both parallel and perpendicular pump and probe pulses, $S_{\|}+2 S_{\perp}$, whereas the anisotropic response is proportional to the difference in the two tensor elements, $S_{\|}$ $S_{\perp} \cdot{ }^{61}$ These combinations of tensor elements are examined to determine whether or not the transition dipole orientations for the charge-transfer resonance are affected by vibrational motions (the individual tensor elements, $S_{\|}$and $S_{\perp}$, are shown in the Supporting Information). Totally symmetric 

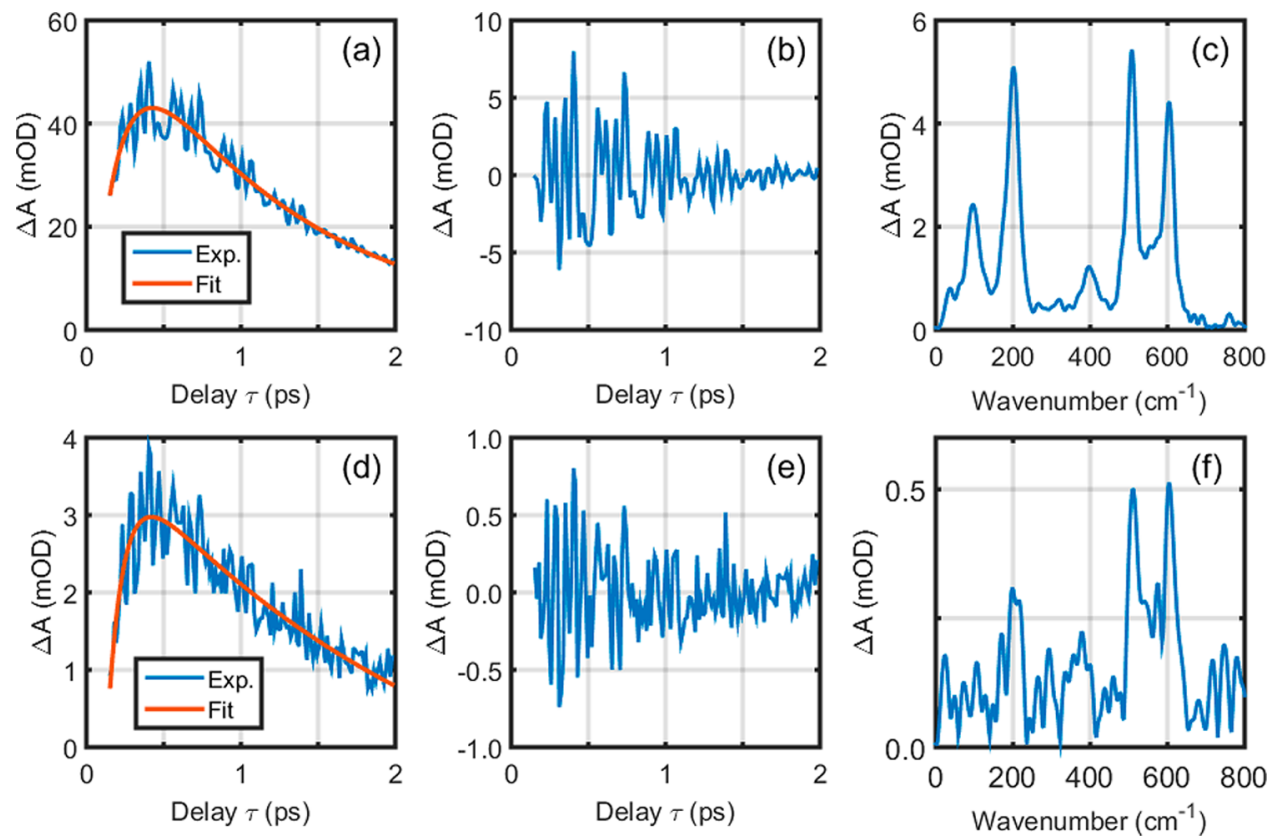

Figure 9. (a) An incoherent baseline is subtracted from the isotropic signal component at $500 \mathrm{~nm}$ to (b) isolate the coherent response. (c) Fourier transformation yields a coherent Raman spectrum for the isotropic HGS signal component. (d) An incoherent baseline is subtracted from the anisotropic signal component at $500 \mathrm{~nm}$ to (e) isolate the coherent response. (f) Fourier transformation yields a coherent Raman spectrum for the anisotropic HGS signal component. All mode frequencies are slightly smaller than those observed with spontaneous Raman spectroscopy, which indicates that the modes are highly populated following back-electron transfer (i.e., the frequencies are lower because of anharmonicity).
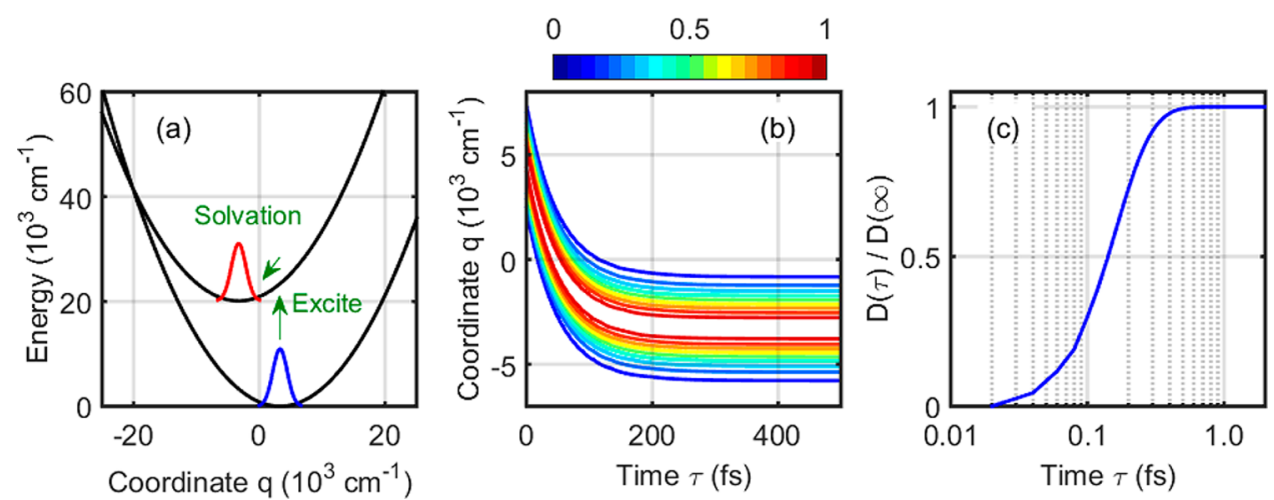

Figure 10. Physical picture suggested by theoretical model. (a) The pump pulse initiates a wavepacket in the collective "solvent" coordinate, which moves toward the point of intersection between potential energy surfaces as $\tau$ increases. Energy is (linearly) mapped onto the collective coordinate for convenience. (b) Motion of the wavepacket, $Y(\tau, q)$, is simulated with eq 24. (c) Growth in the magnitude of the doorway function with $\tau$ represents an increase in the probability of a BET transition. Quantized vibrational modes must promote the BET transition deep within the inverted regime, because the excited-state wavepacket possesses little overlap with the geometry of the transition state in the solvent coordinate.

modes should not affect the transition dipole angles, because they preserve the symmetry of the system, whereas vibrational modes with lower symmetry may cause the transition dipoles to oscillate through a non-Condon effect. ${ }^{62}$

The coherent Raman spectra in Figure 9 show that vibrational resonances associated with the isotropic signal component are 10 times more intense than those found in the anisotropic signal components. We observe resonances corresponding to the 222, 410,538, and $643 \mathrm{~cm}^{-1}$ modes detected with spontaneous Raman spectroscopy. Transient absorption yields resonances at 210,400,510, and $600 \mathrm{~cm}^{-1}$, which suggests that the modes are highly populated following the back-electron-transfer process (i.e., the lower frequencies reflect anharmonicity). Decreases in vibrational frequencies are generally observed in systems, where ground-state recovery is much faster than vibrational cooling. ${ }^{63}$ Relative to the isotropic response, the largest anisotropic intensities are found in the modes at 505 and $610 \mathrm{~cm}^{-1}$, which may reflect weak nonCondon activity (i.e., the transition dipole orientations may be affected by vibrational motion). The $210 \mathrm{~cm}^{-1}$ mode is notably assigned to a totally symmetric stretch involving the ligands and metal center. Thus, a small anisotropic response is anticipated.

IVD. Analysis of the Back-Electron-Transfer Mechanism. Vibrational coherences in the HGS signal component signify contributions from the two paths that end in the upper right of Figure 1. These coherences can originate in either vibrational populations or coherences in the excited electronic state. The model developed in section II can be used to estimate the relative contributions of these pathways. All of the parameters with the exception of the solvation time, $\Lambda^{-1}$, can be extracted from the spectral fitting approach described above. We set $\Lambda^{-1}$ equal to the $75 \mathrm{fs}$, which is reasonable for aqueous 


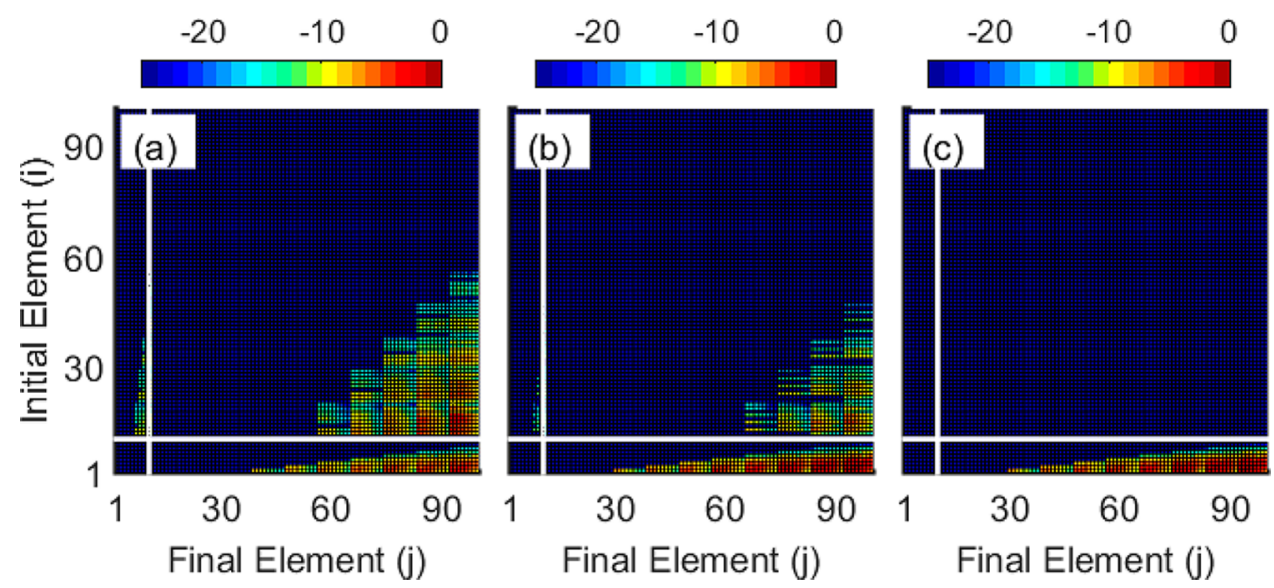

Figure 11. Doorway functions, $D_{i \rightarrow j}(\tau)$, computed with eq 14 and the parameters in Table 1. Only the $222 \mathrm{~cm}^{-1}$ mode is included in these calculations. The times, $\tau$, are (a) 0.2 , (b) 0.5 , and (c) 2 ps. The calculations suggest that vibrational coherences are initiated in the ground electronic state following BET if the system is initially in either a vibrational coherence (upper right) or population (lower right). The behavior transitions from an energy gap-limited regime at $\tau=0.1$ ps to a Franck-Condon-limited regime at $\tau=2$ ps.

solution. ${ }^{36}$ In addition, damping times for the ground $\left(\Gamma_{u l}{ }^{-1}\right)$ and excited $\left(\Gamma_{n k}{ }^{-1}\right)$ states are set equal to $1 \mathrm{ps}$ and $180 \mathrm{fs}$, respectively. The damping times for the excited state are shorter because the lifetimes are limited by BET. It is not necessary to parametrize the product of matrix elements, $\left|\xi_{\mathrm{L}} \mu_{\mathrm{eg}}\right|^{2}\left|V_{\mathrm{eg}}\right|^{2}$, in the doorway function, because normalized signal intensities are analyzed in this section.

We begin by discussing the physical picture associated with the model in Figure 10. Energy has been (linearly) mapped onto the collective coordinate for convenience. ${ }^{14}$ Ground- and excited-state potential energy minima appear at $+\lambda$ and $-\lambda$ for a harmonic bath (and the Stokes shift is $2 \lambda$ ). The point of intersection between free energy surfaces is located at $-\lambda-$ $\left(\Delta G_{\text {eg }}+\lambda\right)^{2} / 4 \lambda$. The wavepacket initiated by the pump pulse can be written as ${ }^{31}$

$$
\begin{aligned}
Y(\tau, q)= & \frac{1}{\alpha(\tau) \sqrt{2 \pi}} \\
& \times \exp \left[-\frac{\left[q+\lambda+\exp (-\Lambda \tau)\left(-\hbar \omega_{\mathrm{L}}-\Delta G_{\mathrm{eg}}-\lambda\right)\right]^{2}}{2 \alpha^{2}(\tau)}\right]
\end{aligned}
$$

Ground-state equilibrium and asymptotic $(Y(\tau \rightarrow \infty, q))$ probability distributions are overlaid on the potential energy surfaces in Figure 10a. The excited-state wavepacket never possesses significant amplitude at the point of intersection during the relaxation process (Figure $10 \mathrm{~b}$ ), because $\left[\mathrm{Ti}\left(\mathrm{cat}_{)_{3}}\right]^{2-}\right.$ is deep within the inverted regime. Nonetheless, the transition probability is large, because displaced intramolecular modes promote transitions across the $20100 \mathrm{~cm}^{-1}$ free energy gap. Motion of the wavepacket in the solvation coordinate is the key physical process responsible for time dependence in the overall magnitude of $D(\tau)$ (i.e., oscillations induced by the vibrational coherences are relatively weak).

Before presenting numerical results, it will be useful to first discuss the three key factors that influence the contributions of various terms to the doorway functions, $D_{n k \rightarrow u l}(\tau)$. The magnitudes of the functions are governed by (i) FranckCondon overlaps for light absorption, (ii) Franck-Condon overlaps for BET, and (iii) the difference between $\Delta G_{\text {eg }}^{0}+\lambda$ and $\hbar\left(\omega_{l}-\omega_{n}\right)$ (see argument of exponential function in eq 15). Because the largest mode displacements in $\left[\mathrm{Ti}(\mathrm{cat})_{3}\right]^{2-}$ are not much greater than 1.0, the terms that make the largest contributions will generally be those in which $n$ and $k$ do not exceed 3. For example, the ratio in Franck-Condon factors for the $0-0$ and $0-3$ vibronic transitions is equal to 0.075 with a mode displacement of 1.24 (i.e., the largest displacement in $\left.\left[\mathrm{Ti}(\mathrm{cat})_{3}\right]^{2-}\right)$. In addition, appreciable Franck-Condon overlaps in the BET process will generally be those in which $l$ and $u$ do not exceed 6, because the product, $\langle n \mid u\rangle\langle k \mid l\rangle$, also appears in eq 15. It follows that the optimal numbers of vibrational quanta $(n, k, l, u)$ become smaller as the mode displacement decreases. The quantity, $\Delta G_{\text {eg }}^{0}+\lambda$, is negative in the inverted regime, so the BET process is accelerated when $\omega_{l} \gg \omega_{n}$. In $\left[\mathrm{Ti}(\text { cat })_{3}\right]^{2-}$, pathways in which $l$ is much larger than a few quanta are energetically favored, because $\Delta G_{\text {eg }}^{0}+\lambda$ is equal to -16810 $\mathrm{cm}^{-1}$ (e.g., $l$ must be close to 11 to conserve energy in a relaxation channel involving the $1482 \mathrm{~cm}^{-1}$ mode). Overall, we suggest that the Franck-Condon overlaps (which favor small $l$ ) and energetic considerations (which favor large $l$ ) compete with each other in $\left[\mathrm{Ti}(\mathrm{cat})_{3}\right]^{2-}$, because the system is deep within the inverted regime and possesses modest mode displacements.

Behaviors of the 222 and $1482 \mathrm{~cm}^{-1}$ modes are compared below to illustrate how Franck-Condon overlaps and energetic constraints influence the weights of various terms in the doorway functions. For convenience, the density matrix elements are shuffled to separate functions associated with different classes of transitions. Indices 1-10 correspond to populations with 0-9 vibrational quanta (the number of quanta is the index minus 1). Indices $11-100$ correspond to coherences. For the coherences, the indices are given by

$$
i=s \cdot n+k-n+\beta \quad\left\{\begin{array}{l}
\beta=0, n<k \\
\beta=1, n>k
\end{array}\right.
$$

and

$$
j=s \cdot l+u-l+\beta \quad\left\{\begin{array}{l}
\beta=0, l<u \\
\beta=1, l>u
\end{array}\right.
$$

where $s$ is the number of states in the basis set (10 in the following calculations). It is useful to consider that the value of $n$ (and $l$ ) is equal to 1 for indices 11-20, 2 for indices 21-30, etc.; the value of $k$ (and $u$ ) cycles from 1 to 10 within each decade. With these rules, population-to-population and coherence-to-coherence transitions appear in the lower left 


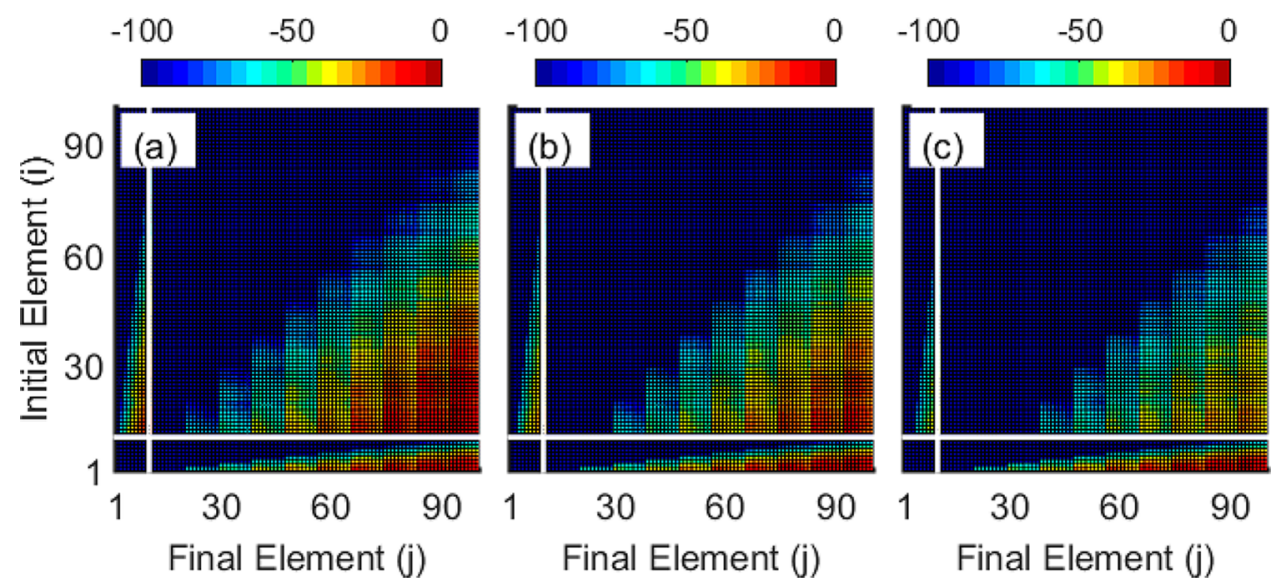

Figure 12. Doorway functions, $D_{i \rightarrow j}(\tau)$, computed with eq 14 and the parameters in Tables 1 and (the mode displacement is taken from Table 2). Only the $1482 \mathrm{~cm}^{-1}$ mode is included in these calculations. The times, $\tau$, are (a) $0.2 \mathrm{ps,} \mathrm{(b)} 0.5 \mathrm{ps}$, and (c) 2 ps. The calculations suggest that vibrational coherences are initiated in the ground electronic state following BET if the system is initially in either a vibrational coherence (upper right) or population (lower right). These calculations show that all pathways will generally be activated in systems with high-frequency $\left(>1000 \mathrm{~cm}^{-1}\right)$ vibrational modes and charge-transfer resonances in the visible spectral range.
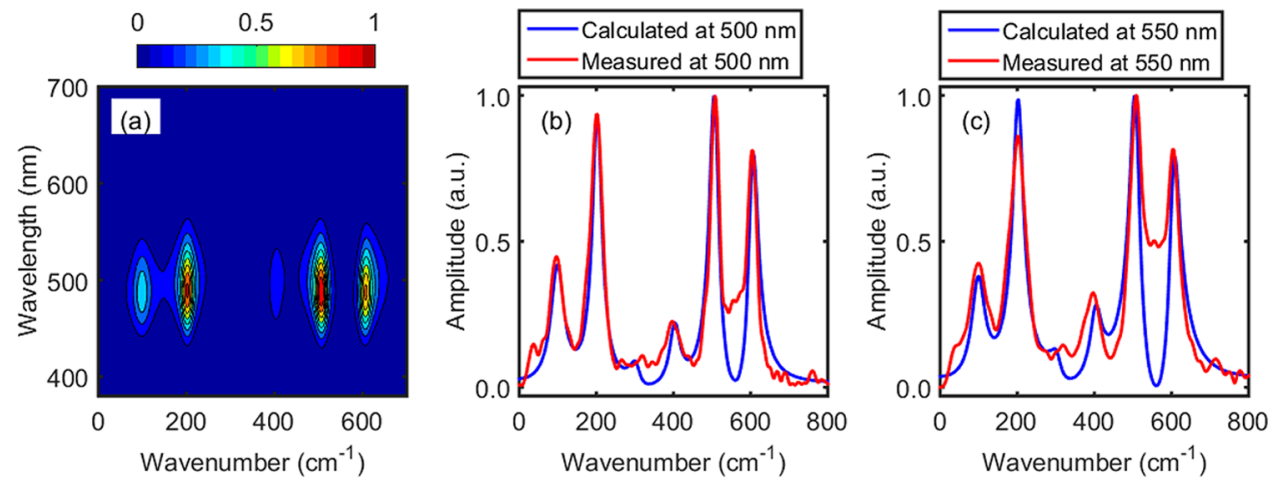

Figure 13. (a) Fourier transform of transient absorption signal calculated with eq 27 and the parameters in Tables 1 and 2. This contour plot can be compared to the measurement in Figure 8. The calculated and measured signals are overlaid at a detection wavelength of (b) $500 \mathrm{~nm}$ and (c) 550 $\mathrm{nm}$. The parameters are adjusted to optimize the fit to the absorbance spectrum (Figure 3), the resonance Raman cross sections (Figure 5), and the coherent part of the transient absorption signal at $500 \mathrm{~nm}$ (Figure 9).

and upper right portions of the matrices in Figures 11 and 12 (white grid lines separate these classes of transitions). Population-to-coherence transitions appear in the lower right.

In Figure 11, the doorway functions for the $222 \mathrm{~cm}^{-1}$ mode exhibit marked changes with time, $\tau$. The dynamics illustrated in Figure 11 indicate that terms in which $n \neq k$ contribute most at early times (i.e., the coherence-to-coherence pathway). With respect to the three aforementioned points, the third point involving the relative magnitudes of $\Delta G_{\mathrm{eg}}^{0}+\lambda$ and $\hbar \omega_{l n}$ is most important at early times, because this is when the energy gap in the solvation coordinate is largest (Figure 10). In this regime, values of $k$ that differ from $n$ (small values of $\langle k \mid m\rangle\langle n \mid m\rangle$ ) are well-tolerated, because large values of $l$ are needed to compensate for the energy gap in the solvation coordinate. At long times $\left(\tau \gg \Lambda^{-1}\right)$, the product of vibrational overlap integrals primarily controls relative contributions of particular terms, and the population-to-coherence pathways dominates (i.e., points $\mathrm{i}$ and ii outcompete point iii). We refer to the marked difference in behaviors at short and long times as representing energy gap-limited and Franck-Condon-limited regimes.

The calculation presented in Figure 12, which involves the $1482 \mathrm{~cm}^{-1}$ mode, is more typical of molecular systems, where the electronic energy gap and mode frequencies do not differ by multiple orders of magnitude. Both coherence-to-coherence and population-to-coherence pathways contribute at times that are both short and long compared to the solvation process. There is only a minor decrease in the relative contribution of the coherence-to-coherence pathway at long times. In comparison with Figure 11, the different behavior exhibited in Figure 12 reflects the higher frequency and smaller displacement of the $1482 \mathrm{~cm}^{-1}$ mode. The higher-mode frequency mitigates the influence of energetic factors, and the smaller displacement enhances the influence of vibrational overlap integrals. Consequently, the decrease in contributions from the coherence-to-coherence pathways with increasing $\tau$ is relatively modest. This time-dependent decrease in coherenceto-coherence pathways would be further suppressed by an increase in the mode displacement. The present calculations suggest that both coherence-to-coherence and population-tocoherence transitions will be prominent in molecular systems with organic moieties, because they generally possess displaced high-frequency $\left(>1000 \mathrm{~cm}^{-1}\right)$ modes and charge-transfer resonances in the visible spectral range. This behavior is consistent with that predicted by Fleming and Jean. ${ }^{30}$

The calculations presented in Figures 11 and 12 illustrate basic principles of the BET mechanism. The next step is to compare experimental and calculated transient absorption 
signals. The vibrational coherences in the HGS signal component, which are of primary interest in this work, are isolated by restricting the summation in the expression for the signal to eliminate the population response. The signal can then be Fourier transformed to obtain the contour plot in Figure 13 .

$$
S\left(\omega_{\tau}, \omega_{\mathrm{det}}\right)=\sum_{\substack{m n k v \\ l \neq u}} \int_{0}^{\infty} D_{n k \rightarrow u l}(\tau) W_{l u v}\left(\tau, \omega_{\mathrm{det}}\right) \exp \left(\mathrm{i} \omega_{\tau} \tau\right)
$$

The calculated signals in Figure 13a can be compared to the measurement in Figure 8. The peak positions and line widths agree fairly well in both dimensions, $\omega_{\tau}$ and $\omega_{\text {det. }}$. The intensities are also in good agreement. In carrying out the fit, we began by assuming the parameters in Table 1 and then introduced changes only when needed to fit the signal acquired with a detection wavelength of $500 \mathrm{~nm}$ (Figure 13b and Table 2). Adjustments are necessary because spontaneous resonance

Table 2. Fitting Parameters for Transient Absorption Signals in Figure 13

\begin{tabular}{ccc}
$\begin{array}{c}\text { vibrational wavenumber } \\
\text { from spontaneous Raman } \\
\text { spectroscopy }\left(\mathrm{cm}^{-1}\right)\end{array}$ & $\begin{array}{c}\text { vibrational wavenumber } \\
\text { from transient absorption } \\
\text { spectroscopy }\left(\mathrm{cm}^{-1}\right)\end{array}$ & $\begin{array}{c}\text { dimensionless } \\
\text { displacement }\end{array}$ \\
222 & 99 & 1.80 \\
538 & 202 & 1.24 \\
643 & 507 & 0.90 \\
1482 & 604 & 0.94 \\
\hline
\end{tabular}

Raman spectroscopy is primarily sensitive to the potential energy surfaces near the Franck-Condon geometry, whereas transient absorption signals reflect a broader range of coordinate space. Minor differences between the fit and measurement are found on the high- and low-frequency sides of the HGS resonance (Figure 13c). We have accounted for time resolution by translating the $40 \mathrm{fs}$ instrument response near $500 \mathrm{~nm}$ into a Gaussian with a fwhm of $520 \mathrm{~cm}^{-1}$. The amount by which a vibrational resonance is attenuated is estimated by the magnitude of the Gaussian function at the respective frequency. ${ }^{14}$

The mode frequencies had to be slightly reduced to fit the transient absorption signals in Figure 13. This is not a surprising observation. A reduction in vibrational frequencies induced by a fast nonradiative transition is usually attributed to anharmonicity. ${ }^{63}$ Third-order "diagonal" force constants (differentiation with respect to individual normal coordinates) computed at the B3LYP/6-311G $(2 \mathrm{~d}, 3 \mathrm{p})$ level for the 538 and $643 \mathrm{~cm}^{-1}$ modes of $\left[\mathrm{Ti}(\mathrm{cat})_{3}\right]^{2-}$ suggest that wavenumber differences between successive vibrational levels reach 507 and $604 \mathrm{~cm}^{-1}$ when the number of vibrational quanta is greater than 50 , which is far in excess of the electronic energy gap (thirdorder derivatives for the 507 and $604 \mathrm{~cm}^{-1}$ modes are 13 and $17(\mathrm{~kg} / \AA) / \mathrm{s}^{2}$, respectively). Therefore, we hypothesize that a large number of off-diagonal, anharmonic force constants (differentiation with respect to mixtures of the vibrational coordinates) combine to reduce the vibrational frequencies from the equilibrium values.

An important distinction should be recognized between the information conveyed by the doorway functions in Figures 11 and 12 and the spectroscopic signals Figure 13. The doorway functions can be thought of as representing the weight of each pathway irrespective of how the probe step is implemented (e.g., visible or mid-infrared light). In fact, the doorway functions assume a mathematical form that is similar to fourthorder "rate functions" developed in earlier work (this similarity will be further discussed in section V). ${ }^{31,35}$ In contrast, the window function effectively filters particular pathways based on vibrational wave function overlap integrals. Thus, the oscillations observed in the signal, which are primarily detected at the fundamental mode frequency, are a somewhat indirect representation of vibronic relaxation channels driven by the BET mechanism.

In summary, the calculations presented in this section have been used to investigate the two BET pathways that lead to vibrational coherences in the ground electronic state. The key conclusions drawn from these calculations are

(i) The most dominant BET transitions involve highfrequency vibrational coherences, $\omega_{l n}$, in the $t_{3}$ interval between perturbative interactions with the donoracceptor coupling (Figure 2).

(ii) The frequencies of promoting modes observed with transient absorption spectroscopy will generally deviate from the predictions of a harmonic model because they will be highly excited following BET (as a consequence of point $\mathrm{i}$ ). This behavior is consistent with other systems that exhibit ultrafast ground-state recovery. ${ }^{63}$

(iii) Model calculations based on empirical parameters suggest that both coherence-to-coherence and population-to-coherence pathways contribute in $\left[\mathrm{Ti}(\mathrm{cat})_{3}\right]^{2-}$ because of its high-frequency modes $\left(>1000 \mathrm{~cm}^{-1}\right)$ and modest displacements.

(iv) The present measurements and simulations demonstrate that pathways ending in the upper right of Figure 1 contribute to the signals. These two pathways cannot be experimentally distinguished without extraordinary time resolution. The only way to distinguish these two pathways is to resolve coherent and incoherent dynamics in the excited state (prior to BET), which has a lifetime with an upper limit of 180 fs (we do not observe contributions from the excited state outside the region of pulse overlap). ${ }^{24}$

\section{BROADER IMPLICATIONS FOR ELECTRON-TRANSFER REACTIONS}

Coherent dynamics in which broadband laser pulses initiate vibrational coherences are not necessarily relevant to applications such as photoelectrochemical cells. ${ }^{64,65}$ For example, the vibrational coherence-to-coherence pathway in Figure 1 requires impulsive excitation and is arguably irrelevant to processes initiated by sunlight. The vibrational populationto-coherence pathway differs from the vibrational coherence-tocoherence pathway in that impulsive excitation is not required. Moreover, according to the model presented in section II, the population-to-coherence pathway may contribute even at times that are long compared to solvation and vibrational dephasing.

The potential implications of this work are more clearly seen if the doorway function is rewritten as a fourth-order rate function. ${ }^{31,35,64}$ In fact, the derivation of the doorway function in section IIB parallels the derivation of the fourth-order rate function for electron transfer presented in earlier work. ${ }^{31}$ The fourth-order rate function can be written as 


$$
K_{n k \rightarrow u l}^{(4)}(\tau)=\left(\frac{\hbar w_{\mathrm{L}}^{2} \sqrt{2\left(\Delta^{2}+w_{\mathrm{L}}^{2}\right)}}{\pi\left|\xi_{\mathrm{L}} \mu_{\mathrm{eg}}\right|^{2}}\right) \cdot D_{n k \rightarrow u l}(\tau)
$$

if the normalization condition of ref 31 is applied. The normalization condition ensures convergence to Marcus' equation at times that are long compared to the solvation time for models with weak vibronic couplings. When $\tau$ is longer than solvation and vibrational dephasing, the fourth-order rate function becomes

$$
\begin{aligned}
K_{n n \rightarrow u l}^{(4)}(\tau \rightarrow \infty)= & \frac{\pi^{1 / 2} \mid V_{\mathrm{eg}}{ }^{2}}{\hbar \sqrt{\lambda k_{\mathrm{B}} T}} B_{m}\langle n \mid m\rangle\langle n \mid m\rangle\langle n \mid u\rangle\langle n \mid l\rangle \\
& \times \exp \left[-\frac{\hbar^{2}\left(\omega_{\mathrm{L}}-\omega_{\mathrm{eg}}\right)^{2}}{4 \lambda k_{\mathrm{B}} T}\right] \\
& \times \exp \left[-\frac{\left(\Delta G_{\mathrm{eg}}^{0}+\lambda-\hbar \omega_{n l}\right)^{2}}{4 \lambda k_{\mathrm{B}} T}\right]
\end{aligned}
$$

where the only surviving terms are those in which $n=k$. In other words, the coherence-to-coherence pathway, $n k \rightarrow u l$, vanishes when $\tau$ is large but the population-to-coherence pathway, $n n \rightarrow u l$, survives.

This idea can be taken a step further if we let the system establish a Boltzmann distribution of vibrational populations in the excited state before BET. In our notation, the traditional second-order (population-to-population) expression for the BET process can be written as ${ }^{17}$

$$
\begin{aligned}
K_{n n \rightarrow m m}^{(2)}= & \frac{\pi^{1 / 2}\left|V_{\mathrm{eg}}\right|^{2}}{\hbar \sqrt{\lambda k_{\mathrm{B}} T}} \sum_{n m} B_{m}|\langle n \mid m\rangle|^{2} \\
& \times \exp \left[\frac{-\left(\Delta G+\lambda-\hbar \omega_{n m}\right)^{2}}{4 \lambda k_{\mathrm{B}} T}\right]
\end{aligned}
$$

Vibrational coherences are not initiated by BET at this level of theory, because the coherences do not survive the trace over all quantum states taken in the evaluation of the correlation function. ${ }^{15}$ We question whether or not elimination of the population-to-coherence pathway by a trace at second order is fully justified. Restoration of a Boltzmann distribution of vibrational quanta occurs rapidly following BET because of intramolecular vibrational cooling and solute-to-solvent vibrational energy transfer. These processes generally occur on a time scale comparable to picosecond vibrational dephasing. Thus, it is reasonable to describe the sequence of BET and vibrational cooling as a higher-order perturbative process. For example, the two donor-acceptor interactions associated with BET could be followed by one perturbative interaction with a component of the Hamiltonian associated with solute-solvent interactions, thereby producing a population that survives the trace over quantum states (Figure 14).

Because a detailed description of vibrational cooling dynamics is beyond the scope of this work, we suggest that it is instructive to simply take the trace over the electronic states in writing an ad hoc second-order rate formula for BET processes that begin with an equilibrium condition. Equation 30 can be rewritten as

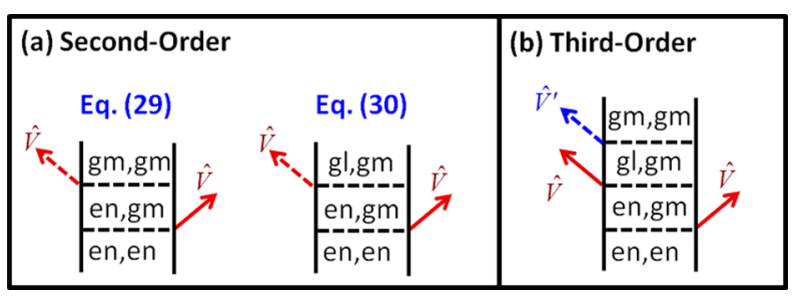

Figure 14. We consider whether or not population-to-coherence pathways are relevant in systems regardless of the electron-transfer time scale. (a) Only population-to-population transitions occur in the traditional second order rate formula, eq 30 . The population-tocoherence pathway survives if the trace is taken only over electronic states in eq 31. (b) In a higher-order model, a trace over all quantum states can be carried out if subsequent vibrational cooling dynamics are accounted for. Vibrational population-to-coherence transitions may then contribute regardless of the electron-transfer rate. The operators $\hat{V}$ and $\hat{V}^{\prime}$ denote the donor-acceptor coupling and the solute-solvent interaction, respectively.

$$
\begin{aligned}
K_{n n \rightarrow l m}^{(2)}= & \frac{\pi^{1 / 2}\left|V_{\mathrm{eg}}\right|^{2}}{\hbar \sqrt{\lambda k_{\mathrm{B}} T}} \sum_{m n l} B_{n}\langle n \mid m\rangle\langle n \mid l\rangle \\
& \times \exp \left[\frac{-\left(\Delta G+\lambda-\hbar \omega_{n m}\right)^{2}}{4 \lambda k_{\mathrm{B}} T}\right]
\end{aligned}
$$

if we let the electron-transfer transition terminate in a vibrational coherence. Feynman diagrams associated with eqs 30 and 31 are given in Figure 14a. The diagrams take only the interactions with the donor-acceptor coupling into account. The larger number of pathways found in $K_{n n \rightarrow l m}^{(2)}$ will generally enhance the overall rate. For example, the ratio, $K_{n n \rightarrow l m}^{(2)} / K_{n n \rightarrow m m}^{(2)}$, is equal to 25 for a system with the 4 modes accounted for in Table 2 when the summations are taken over the 20 lowestenergy states. This ratio will become even larger when additional intramolecular modes are taken into account. The problem of ending the $K_{n n \rightarrow l m}^{(2)}$ pathway in Figure 14a in vibrational coherence can be remedied by incorporating the perturbative part of the Hamiltonian that induces vibrational cooling (Figure 14b). Only one additional interaction is required to convert the system from a coherence to a population. Thus, a third-order rate function can be developed to describe this type of process.

\section{CONCLUDING REMARKS}

In summary, optical spectroscopies and model calculations have been used to investigate contributions of nonequilibrium BET pathways in the transition metal complex, $\left[\mathrm{Ti}(\mathrm{cat})_{3}\right]^{2-}$. Vibrational coherences observed in transient absorption experiments signify pathways that end in the upper right of Figure 1. Our empirical model suggests that both vibrational population-to-coherence and vibrational coherence-to coherence pathways make significant contributions in $\left[\mathrm{Ti}(\mathrm{cat})_{3}\right]^{2-}$ and other systems with high-frequency modes $\left(>1000 \mathrm{~cm}^{-1}\right)$ and charge-transfer resonances in the visible spectrum. In principle, the two types of pathways can be experimentally distinguished with extraordinary time resolution. In such measurements, vibrational populations and coherences in the excited electronic state can be correlated to the vibrational coherences in the ground electronic state. A key fundamental limitation is that the lifetime must be longer than the period of the vibrational mode. This is problematic because wavepacket motions in the ground state can only be initiated when BET is 

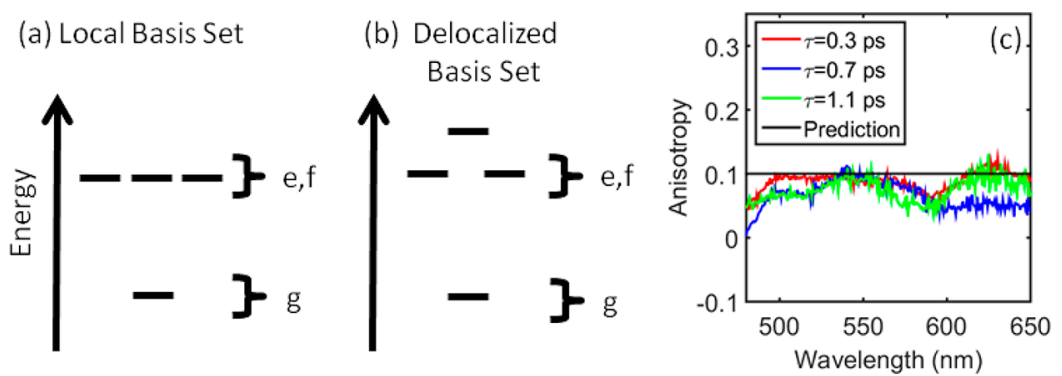

Figure 15. Electronic structure viewed in (a) localized and (b) delocalized basis sets. (c) The transient absorption anisotropy associated with the HGS signal component agrees well with the prediction of 0.1 , which is independent of the basis set. The uncertainty in the anisotropy is approximately 0.05 . The anisotropy in the HGS signal component should not be used to argue for one basis set or the other.

ultrafast (e.g., phase coherence will otherwise be lost in the ensemble). An important practical limitation is that unambiguous information cannot be obtained when the pump and probe pulses are overlapped in the sample. We estimate that the two pathways can be experimentally distinguished with continuum pulses that are compressed to $10 \mathrm{fs}$ or less.

The broader implication of this work is that contributions from the coherent pathways in Figure 1 are generally not negligible if electron transfer is faster than solvation and vibrational dephasing. Such subpicosecond electron-transfer processes are fairly common at molecule-semiconductor interfaces in photoelectrochemical cells. ${ }^{64,65}$ Therefore, a better understanding of these pathways will be useful for interpreting ultrafast spectroscopy measurements in these systems. ${ }^{12,64,66-69}$ In this work, parameters derived from spontaneous Raman experiments were used to simulate transient absorption signals; however, this parametrization scheme may fail in systems that undergo large excited-state geometry changes. Femtosecond stimulated Raman scattering methods ${ }^{70,71}$ or two-dimensional electronic-vibrational spectroscopy ${ }^{72,73}$ may be used to investigate intramolecular motions at nonequilibrium geometries in such systems. It will also be helpful to integrate molecular dynamics simulations into the description.

We question whether or not vibrational population-tocoherence transients are relevant to electron-transfer processes regardless of their time scales. Observations based on ultrafast spectroscopies are limited to BET dynamics that are faster than vibrational dephasing, because vibrational motions can only be detected if a macroscopic coherence is preserved. However, it will be interesting to consider the relevance of vibrational coherences initiated from quasi-equilibrium initial conditions as suggested by eq 31 . These pathways do not survive the trace over electronic and vibrational quantum states at second-order in perturbation theory but can contribute in higher-order descriptions that take vibrational cooling processes into account.

\section{APPENDIX: ANALYSIS OF HGS ANISOTROPY IN LOCALIZED AND DELOCALIZED BASIS SETS}

Isotropic and anisotropic vibrational spectra are compared in Figure 9 to explore the possibility of non-Condon effects. Here, we show that the overall magnitude of the anisotropy in the HGS signal component should not be used to establish the most appropriate basis set in which to view the electronic structure (i.e., localized versus delocalized). The electronic structures of both localized (uncoupled) and delocalized (coupled) basis sets are presented in Figure 15. In both cases, there is a ground state and three singly-excited states related to the ligand-to-metal charge-transfer transitions. All three transition dipoles are equal in the uncoupled basis set, whereas the coupled basis set possesses two allowed transitions (with equal transition dipoles) and one higher-energy, forbidden transition (Supporting Information).

For these systems, signal strengths with parallel and perpendicular pump and probe polarizations can be written as ${ }^{74-76}$

$$
S_{\|}=\frac{|\mu|^{4}}{15} \sum_{a=1}^{N} \sum_{b=1}^{N}\left[1+2 \cos ^{2}\left(\theta_{a, b}\right)\right]
$$

and

$$
S_{\perp}=\frac{|\mu|^{4}}{30} \sum_{a=1}^{N} \sum_{b=1}^{N}\left[4-2 \cos ^{2}\left(\theta_{a, b}\right)\right]
$$

where $N$ is the number of optically-allowed transitions in the respective basis set. The transition dipoles, $\mu$, are equal for each allowed transition in both basis sets, so they have been moved in front of the summations (i.e., only relative magnitudes of the two tensor elements are relevant).

For the localized (uncoupled) basis set, the parallel and perpendicular signal strengths are $S_{\|}=6 / 15$ and $S_{\|}=9 / 30(N$ $=3$ and $\left.\theta_{a, b}=60^{\circ}\right)$. The transition dipoles have been removed from the magnitude of each tensor element, because they are common to both formulas. The parallel and perpendicular signal strengths are $S_{\|}=4 / 15$ and $S_{\|}=3 / 15$ for the delocalized (coupled) basis set $\left(N=2\right.$ and $\left.\theta_{a, b}=90^{\circ}\right)$. For both basis sets, we then obtain an anisotropy of

$$
r=\frac{S_{\|}-S_{\perp}}{S_{\|}+2 S_{\perp}}=0.1
$$

The experimental anisotropy for the HGS signal component is displayed at three delay times in Figure 15c. The earliest delay time is $300 \mathrm{fs}$, which is longer than the $180 \mathrm{fs}$ lifetime of the excited state. Therefore, excited-state emission and excited-state absorption are negligible in each measurement. The agreement between experiment and theory demonstrated in Figure 15 is strong evidence that measurements with polarized laser pulses are interpreted correctly. The uncertainty in the anisotropy is approximately 0.05 .

The delocalized basis set is justified when the interligand coupling strength is large compared to the magnitudes of the fluctuations in energy levels, whereas the localized basis set corresponds to the opposite limit. Recent work describes how these basic aspects of electronic structures in condensed phases, which are well-established in molecular aggregates, ${ }^{6,45-48}$ generalize to transition metal complexes. ${ }^{49,50}$ Time-dependent density functional theory calculations conducted at the B3LYP/ 
6-311G(2d,3p) level yield an interligand coupling strength of $642 \mathrm{~cm}^{-1}$ (Supporting Information), which is comparable to the empirical thermal fluctuation magnitude $1150 \mathrm{~cm}^{-1}$ (eq 5). This suggests that $\left[\mathrm{Ti}(\mathrm{cat})_{3}\right]^{2-}$ resides in an intermediate regime, where valid arguments can be made for either basis set. The analysis presented in this section shows that such arguments are irrelevant to the magnitudes of tensor elements acquired with parallel and perpendicular pump and probe polarizations. The preferred choice of basis set is not important in the present study, which is concerned with establishing the coherent relaxation pathways in Figure 1.

\section{ASSOCIATED CONTENT}

\section{S Supporting Information}

The Supporting Information is available free of charge on the ACS Publications website at DOI: 10.1021/acs.jpca.6b04313.

Additional details regarding the decomposition of transient absorption signals, discussion of the choice of basis in the context of time-dependent density function theory calculations (PDF)

\section{AUTHOR INFORMATION}

\section{Corresponding Author}

*A. M. Moran. E-mail: ammoran@email.unc.edu. Telephone: 919-962-0289.

\section{Notes}

The authors declare no competing financial interest.

\section{ACKNOWLEDGMENTS}

This work was primarily funded by the UNC Energy Frontier Research Center (EFRC) "Center for Solar Fuels", an EFRC funded by the U.S. Department of Energy, Office of Science, Office of Basic Energy Sciences under award DE-SC0001011 (T.P.C., P.G.G., and Z.G.). W.Y. and D.J.D. acknowledge support from the Office of Naval Research (Grant No. N000141410221). EFRC support was used to conduct spectroscopic experiments and develop the theoretical model. W.Y. and D.J.D. synthesized $\left[\mathrm{Ti}(\mathrm{cat})_{3}\right]^{2-}$ with support from the Office of Naval Research (Grant No. N000141410221).

\section{REFERENCES}

(1) Vos, M. H.; Lambry, J.-C.; Robles, S. J.; Youvan, D. C.; Breton, J.; Martin, J.-L. Direct Observation of Vibrational Coherence in Bacterial Reaction Centers Using Femtosecond Absorption Spectroscopy. Proc. Natl. Acad. Sci. U. S. A. 1991, 88, 8885-8889.

(2) Vos, M. H.; Rappaport, F.; Lambry, J.-C.; Breton, J.; Martin, J.-L. Visualization of Coherent Nuclear Motion in a Membrane Protein by Femtosecond Spectroscopy. Nature 1993, 363, 320-325.

(3) Peteanu, L. A.; Schoenlein, R. W.; Wang, H.; Mathies, R. A.; Shank, C. V. The First Step in Vision Occurs in Femtoseconds: Complete Blue and Red Spectral Studies. Proc. Natl. Acad. Sci. U. S. A. 1993, 90, 11762-11766.

(4) Banin, U.; Kosloff, R.; Ruhman, S. Femtosecond Chemical Dynamics in Solution: Photodissociation of $\mathrm{I}_{3}^{-}$. Isr. J. Chem. 1993, 33, 141-156.

(5) Zhu, L.; Sage, J. T.; Champion, P. M. Observation of Coherent Reaction Dynamics in Heme Proteins. Science 1994, 266, 629-632.

(6) Wynne, K.; Reid, G. D.; Hochstrasser, R. M. Vibrational Coherence in Electron Transfer: The Tetracyanoethylene-Pyrene Complex. J. Chem. Phys. 1996, 105, 2287-2297.

(7) Spörlein, S.; Zinth, W.; Wachtveitl, J. Vibrational Coherence in Photosynthetic Reaction Centers Observed in the Bacteriochlorophyll Anion Band. J. Phys. Chem. B 1998, 102, 7492-7496.
(8) Arnett, D. C.; Moser, C. C.; Dutton, P. L.; Scherer, N. F. The First Events in Photosynthesis: Electronic Coupling and Energy Transfer Dynamics in the Photosynthetic Reaction Center from Rhodobacter sphaeroides. J. Phys. Chem. B 1999, 103, 2014-2032.

(9) Lynch, M. S.; Van Kuiken, B. E.; Daifuku, S. L.; Khalil, M. On the Role of High-Frequency Intramolecular Vibrations in Ultrafast BackElectron Transfer Reactions. J. Phys. Chem. Lett. 2011, 2, 2252-2257.

(10) Fuller, F. D.; Pan, J.; Gelzinis, A.; Butkus, V.; Senlik, S. S.; Wilcox, D. E.; Yocum, C. F.; Valkunas, L.; Abramavicius, D.; Ogilvie, J. P. Vibronic Coherence in Oxygenic Photosynthesis. Nat. Chem. 2014, 6, 706-711.

(11) Song, Y.; Clafton, S. N.; Pensack, R. D.; Kee, T. W.; Scholes, G. D. Vibrational Coherence Probes the Mechanism of Ultrafast Electron Transfer in Polymer-Fullerene Blends. Nat. Commun. 2014, 5, 4933.

(12) Tisdale, W. A.; Williams, K. J.; Timp, B. A.; Norris, D. J.; Aydil, E. S.; Zhu, X.-Y. Hot-Electron Transfer from Semiconductor Nanocrystals. Science 2010, 328, 1543-1547.

(13) Huber, R.; Dworak, L.; Moser, J. E.; Grätzel, J.; Wachtveitl, J. Beyond Vibrationally Mediated Electron Transfer: Coherent Phenomena Induced by Ultrafast Charge Separation. J. Phys. Chem. C 2016, 120,8534

(14) Mukamel, S. Principles of Nonlinear Optical Spectroscopy; Oxford University Press: New York, 1995.

(15) Nitzan, A. Chemical Dynamics in Condensed Phases; Oxford University Press: Oxford, U.K., 2006.

(16) Valkunas, L.; Abramavicius, D.; Mančal, T. Molecular Excitation Dynamics and Relaxation: Quantum Theory and Spectroscopy; WileyVCH: Weinheim, 2013.

(17) Barbara, P. F.; Meyer, T. J.; Ratner, M. A. Contemporary Issues in Electron Transfer Research. J. Phys. Chem. 1996, 100, 1314813168.

(18) Bixon, M.; Jortner, J. Vibrational Coherence in Nonadiabatic Dynamics. J. Chem. Phys. 1997, 107, 1470-1482.

(19) Clarke, T. M.; Durrant, J. R. Charge Photogeneration in Organic Solar Cells. Chem. Rev. 2010, 110, 6736-6767.

(20) Ross, R. T.; Nozik, A. J. Efficiency of Hot-Carrier Solar Energy Converters. J. Appl. Phys. 1982, 53, 3813-3818.

(21) Jin, X.; Li, Y.; Chen, Z.; Wei, T.-H.; He, X.; Sun, W. Energy Level Control: Toward an Efficient Hot Electron Transport. Sci. Rep. 2014, 4, 5983.

(22) Li, L.; Kanai, Y. Excited Electron Dynamics at SemiconductorMolecule Type-II Heterojunction Interface: First-Principles Dynamics Simulation. J. Phys. Chem. Lett. 2016, 7, 1495-1500.

(23) Borgias, B. A.; Cooper, S. R.; Koh, Y. B.; Raymond, K. N. Synthetic, Structural, and Physical Studies of Titanium Complexes of Catechol and 3,5-tert-butylcatechol. Inorg. Chem. 1984, 23, 10091016.

(24) Wang, Y.; Hang, K.; Anderson, N. A.; Lian, T. Comparison of Electron Transfer Dynamics in Molecule-to-Nanoparticle and Intramolecular Charge Transfer Complexes. J. Phys. Chem. B 2003, 107, 9434-9440.

(25) Hush, N. S. Homogeneous and Heterogeneous Optical and Thermal Electron Transfer. Electrochim. Acta 1968, 13, 1005-1023.

(26) Yan, Y. J.; Sparpaglione, M.; Mukamel, S. Solvation Dynamics in Electron-Transfer, Isomerization, and Nonlinear Optical Processes. A Unified Liouville-Space Theory. J. Phys. Chem. 1988, 92, 4842-4853.

(27) Britt, B. M.; McHale, J. L.; Friedrich, D. M. Application of Time Dependent Raman Theory to Raman Excitation Profiles of Hexamethylbenzene: Tetracyanoethylene Electron Donor-Acceptor Complex. J. Phys. Chem. 1995, 99, 6347-6355.

(28) Kelley, A. M. Resonance Raman Intensity Analysis of Vibrational and Solvent Reorganization in Photoinduced Charge Transfer. J. Phys. Chem. A 1999, 103, 6891-6903.

(29) Hupp, J. T.; Williams, R. D. Using Resonance Raman Spectroscopy to Examine Vibrational Barriers to Electron Transfer and Electronic Delocalization. Acc. Chem. Res. 2001, 34, 808-817.

(30) Jean, J. M.; Fleming, G. R. Competition Between Energy and Phase Relaxation in Electronic Curve Crossing Processes. J. Chem. Phys. 1995, 103, 2092-2101. 
(31) Li, L.; Giokas, P. G.; Kanai, Y.; Moran, A. M. Modeling TimeCoincident Ultrafast Electron Transfer and Solvation Processes at Molecule-Semiconductor Interfaces. J. Chem. Phys. 2014, 140, 234109.

(32) Coalson, R. D.; Evans, D. G.; Nitzan, A. A Nonequlibrium Golden Rule Formula for Electronic State Populations in Nonadiabatically Coupled Systems. J. Chem. Phys. 1994, 101, 436-448.

(33) Strodel, B.; Stock, G. Quantum Modeling of Transient Infrared Spectra Reflecting Photoinduced Electron-Transfer Dynamics. J. Chem. Phys. 2006, 124, 114105.

(34) Baiz, C. R.; Kubarych, K. J.; Geva, E. Molecular Theory and Simulation of Coherence Transfer in Metal Carbonyls and Its Signature on Multidimensional Infrared Spectra. J. Phys. Chem. B 2011, 115, 5322-5339.

(35) Molesky, B. P.; Moran, A. M. Fourth-Order Perturbative Model for Photoinduced Internal Conversion Processes. J. Phys. Chem. A 2013, 117, 13954-13966.

(36) Horng, M. L.; Gardecki, J. A.; Papazyan, A.; Maroncelli, M. Subpicosecond Measurements of Polar Solvation Dynamics: Coumarin 153 Revisited. J. Phys. Chem. 1995, 99, 17311-17337.

(37) Myers, A. B.; Mathies, R. A.; Tannor, D. J.; Heller, E. J. Excited State Geometry Changes from Preresonance Raman Intensities: Isoprene and Hexatriene. J. Chem. Phys. 1982, 77, 3857-3866.

(38) Kwac, K.; Cho, M. Two-Color Pump-Probe Spectroscopies of Two- and Three-Level Systems: 2-Dimensional Line Shapes and Solvation Dynamics. J. Phys. Chem. A 2003, 107, 5903-5912.

(39) Myers, A. B.; Mathies, R. A. Resonance Raman intensities: a probe of excited state structures and dynamics. In Biological Applications of Raman Spectroscopy; Spiro, T. G., Ed.; Wiley: New York, 1987; Vol. 2, pp 1.

(40) Myers, A. B. Excited Electronic State Properties From GroundState Resonance Raman Intensities. In Laser Techniques in Chemistry; Myers, A. B., Rizzo, T. R., Eds.; John Wiley \& Sons: New York, 1995; Vol. 23, pp 325-384.

(41) Damrauer, N. H.; Cerullo, G.; Yeh, A.; Boussie, T. R.; Shank, C. V.; McCusker, J. K. Femtosecond Dynamics of Excited-State Evolution in $\left[\mathrm{Ru}(\mathrm{bpy})_{3}\right]^{2+}$. Science 1997, 275, 54-57.

(42) Yeh, A.; Shank, C. V.; McCusker, J. K. Ultrafast Electron Localization Dynamics Following Photo-Induced Charge Transfer. Science 2000, 289, 935-938.

(43) Shaw, G. B.; Brown, C. L.; Papanikolas, J. M. Investigation of Interligand Electron Transfer in Polypyridyl Complexes of Os(II) Using Femtosecond Polarization Anisotropy Methods: Examination of Os(bpy) ${ }_{3}^{2+}$. J. Phys. Chem. A 2002, 106, 1483-1495.

(44) Stark, C. W.; Schreier, W. J.; Lucon, J.; Edwards, E.; Douglas, T.; Kohler, B. Interligand Electron Transfer in Heteroleptic Ruthenium(II) Complexes Occurs on Multiple Time Scales. J. Phys. Chem. A 2015, 119, 4813-4824.

(45) Davydov, A. Theory of Molecular Excitons; Plenum: New York, 1971.

(46) van Amerongen, H.; Valkunas, L.; van Grondelle, R. Photosynthetic Excitons; World Scientific: Singapore, 2000.

(47) Spano, F. C. Excitons in Conjugated Oligomer Aggregates, Films, and Crystals. Annu. Rev. Phys. Chem. 2006, 57, 217-243.

(48) Abramavicius, D.; Palmieri, B.; Voronine, D. V.; Sanda, F.; Mukamel, S. Coherent Multidimensional Optical Spectroscopy of Excitons in Molecular Aggregates; Quasiparticle versus Supermolecule Perspectives. Chem. Rev. 2009, 109, 2350-2408.

(49) Miller, S. A.; Moran, A. M. Nonlinear Optical Detection of Electron Transfer Adiabaticity in Metal Polypyridyl Complexes. J. Phys. Chem. A 2010, 114, 2117-2126.

(50) Albert, V. V.; Badaeva, E.; Kilina, S.; Sykora, M.; S, T. The Frenkel Exciton Hamiltonian for Functionalized Ru(II)-bpy Complexes. J. Lumin. 2011, 131, 1739-1746.

(51) Moran, A. M.; Delbecque, C. Solvent Effects on Ground and Excited Electronic State Structures of the Push-Pull Chromophore Julolidinyl-n-N,N'-diethylthiobarbituric Acid. J. Phys. Chem. A 2001, 105, 10208-10219.
(52) Moran, A. M.; Kelley, A. M. Solvent effects on ground and excited electronic state structures of p-nitroaniline. J. Chem. Phys. 2001, 115, 912-924.

(53) Dattelbaum, D. M.; Kober, E. M.; Papanikolas, J. M.; Meyer, T. J. Application of time-resolved near-infrared spectroscopy (TRNIR) to the metal-to-ligand charge transfer (MLCT) excited state(s) of $\left[\mathrm{Os}(\mathrm{phen})_{3}\right]^{2+}$. Chem. Phys. 2006, 326, 71-78.

(54) Shriver, D. F.; Dunn, J. B. R. The Backscattering Geometry for Raman Spectroscopy of Colored Materials. Appl. Spectrosc. 1974, 28, 319-325.

(55) Dudik, J. M.; Johnson, C. R.; Asher, S. A. Wavelength Dependence of the Preresonance Raman Cross Sections of $\mathrm{CH}_{3} \mathrm{CN}$, $\mathrm{SO}_{4}{ }^{2-}, \mathrm{ClO}_{4}{ }^{-}$, and $\mathrm{NO}_{3}{ }^{-}$. J. Chem. Phys. 1985, 82, 1732-1740.

(56) Frisch, M. J.; Trucks, G. W.; Schlegel, H. B.; Scuseria, G. E.; Robb, M. A.; Cheeseman, J. R.; Scalmani, G.; Barone, V.; Mennucci, B.; Petersson, G. A.; et al. Gaussian 09; Gaussian Inc.: Wallingford, CT, 2009.

(57) Lilichenko, M.; Kelley, A. M. Application of Artificial Neural Networks and Genetic Algorithms to Modeling Molecular Electronic Spectra in Solution. J. Chem. Phys. 2001, 114, 7094-7102.

(58) Kovalenko, S. A.; Schanz, R.; Hennig, H.; Ernsting, N. P. Cooling Dynamics of an Optically Excited Molecular Probe in Solution from Femtosecond Broadband Transient Absorption Spectroscopy. J. Chem. Phys. 2001, 115, 3256-3273.

(59) Pecourt, J.-M.; Peon, J.; Kohler, B. DNA Excited-State Dynamics: Ultrafast Internal Conversion and Vibrational Cooling in a Series of Nucleosides. J. Am. Chem. Soc. 2001, 123, 10370-10378.

(60) Champion, P. M.; Rosca, F.; Ionascu, D.; Cao, W.; Ye, X. Rapid Timescale Processes and the Role of Electronic Surface Coupling in the Photolysis of Diatomic Ligands from Heme Proteins. Faraday Discuss. 2004, 127, 123-135.

(61) Vöhringer, P.; Scherer, N. F. Transient Grating Optical Heterodyne Detected Impulsive Stimulated Raman Scattering in Simple Liquids. J. Phys. Chem. 1995, 99, 2684-2695.

(62) Womick, J. M.; West, B. A.; Scherer, N. F.; Moran, A. M. Vibronic Effects in the Spectroscopy and Dynamics of C-Phycocyanin. J. Phys. B: At., Mol. Opt. Phys. 2012, 45, 154016.

(63) Schrader, T.; Sieg, A.; Koller, F.; Schereier, W.; An, Q.; Zinth, W.; Gilch, P. Vibrational Relaxation Following Ultrafast Internal Conversion: Comparing IR and Raman Probing. Chem. Phys. Lett. 2004, 392, 358-364.

(64) Ardo, S.; Meyer, G. J. Photodriven Heterogeneous Charge Transfer with Transition-Metal Compounds Anchored to $\mathrm{TiO}_{2}$ Semiconductor Surfaces. Chem. Soc. Rev. 2009, 38, 115-164.

(65) Concepcion, J. J.; Jurss, J.; Brennaman, M. K.; Hoertz, P. G.; Patrocinio, A. O. T.; Iha, N. Y. M.; Templeton, J. L.; Meyer, T. J. Making Oxygen with Ruthenium Complexes. Acc. Chem. Res. 2009, 42, $1954-1965$

(66) Zimmermann, C.; Willig, F.; Ramakrishna, S.; Burnfeindt, B.; Pettinger, B.; Eichberger, R.; Storck, W. Experimental Fingerprints of Vibrational Wave-Packet Motion during Ultrafast Heterogeneous Electron Transfer. J. Phys. Chem. B 2001, 105, 9245-9253.

(67) Asbury, J. B.; Hao, E.; Wang, Y.; Ghosh, H. N.; Lian, T. Ultrafast Electron Transfer Dynamics from Molecular Adsorbates to Semiconductor Nanocrystalline Thin Films. J. Phys. Chem. B 2001, 105, $4545-4557$

(68) Anderson, N. A.; Lian, T. Ultrafast Electron Transfer at the Molecule-Semiconductor Nanoparticle Interface. Annu. Rev. Phys. Chem. 2005, 56, 491-519.

(69) Duncan, W. R.; Prezhdo, O. V. Theoretical Studies of Photoinduced Electron Transfer in Dye-Sensitized $\mathrm{TiO}_{2}$. Annu. Rev. Phys. Chem. 2007, 58, 143-184.

(70) McCamant, D. W.; Kukura, P.; Yoon, S.; Mathies, R. A Femtosecond Broadband Stimulated Raman Spectroscopy: Apparatus and Methods. Rev. Sci. Instrum. 2004, 75, 4971-4980.

(71) Kukura, P.; McCamant, D. W.; Mathies, R. A. Femtosecond stimulated Raman spectroscopy. Annu. Rev. Phys. Chem. 2007, 58, 461-488. 
(72) Oliver, T. A. A.; Lewis, N. H. C.; Fleming, G. R. Correlating the Motion of Electrons and Nuclei with Two-Dimensional ElectronicVibrational Spectroscopy. Proc. Natl. Acad. Sci. U. S. A. 2014, 111, 10061-10066.

(73) Courtney, T. L.; Fox, Z. W.; Estergreen, L.; Khalil, M. Measuring Coherently Coupled Intramolecular Vibrational and Charge-Transfer Dynamics with Two-Dimensional VibrationalElectronic Spectroscopy. J. Phys. Chem. Lett. 2015, 6, 1286-1292.

(74) Andrews, D. L.; Thirunamachandran, T. On three-dimensional rotational averages. J. Chem. Phys. 1977, 67, 5026-5033.

(75) Hochstrasser, R. M. Two-dimensional IR-spectroscopy: polarization anisotropy effects. Chem. Phys. 2001, 266, 273-284.

(76) Dreyer, J.; Moran, A. M.; Mukamel, S. Tensor Components in Three Pulse VIbrational Echoes of a Rigid Dipeptide. Bull. Korean Chem. Soc. 2003, 24, 1091-1096. 